\title{
The Effect of Beta-Blockers on Hemodynamic Parameters in Patient-Specific Blood Flow Simulations of Type-B Aortic Dissection: An Virtual Study
}

\author{
Mohammad Amin Abazari \\ K.N.Toosi University of Technology \\ Deniz Rafieianzab \\ K.N.Toosi University of Technology \\ M. Soltani \\ University of Waterloo \\ Mona Alimohammadi ( $\square$ mona@alimohammadi.co.uk) \\ K.N.Toosi University of Technology
}

\section{Research Article}

Keywords: Type-B aortic dissection, Patient-specific, Computational fluid dynamic (CFD), Windkessel model, Anti impulse therapy

Posted Date: March 2nd, 2021

DOI: https://doi.org/10.21203/rs.3.rs-255605/v1

License: (c) (i) This work is licensed under a Creative Commons Attribution 4.0 International License. Read Full License

Version of Record: A version of this preprint was published at Scientific Reports on August 6th, 2021. See the published version at https://doi.org/10.1038/s41598-021-95315-w. 


\section{The Effect of Beta-Blockers on Hemodynamic Parameters in Patient-Specific Blood Flow Simulations of Type-B Aortic Dissection: An Virtual Study}

\section{Mohammad Amin Abazari ${ }^{a}$, Deniz Rafieianzab ${ }^{a}$, M. Soltani ${ }^{a, b, c, d, e}$, Mona Alimohammadi, ${ }^{\mathrm{a}, *}$}

${ }^{a}$ Department of Mechanical Engineering, K. N. Toosi University of Technology, Tehran, Iran

${ }^{b}$ Department of Electrical and Computer Engineering, Faculty of Engineering, School of Optometry and Vision Science, Faculty of Science, University of Waterloo, Waterloo, Canada

c Advanced Bio Initiative Center, Multidisciplinary International Complex, K. N. Toosi University of Technology, Tehran, Iran

${ }^{\mathrm{d}}$ Centre for Biotechnology and Bioengineering (CBB), University of Waterloo, Waterloo, ON, Canada

${ }^{\mathrm{e}}$ Cancer Biology Research Center, Cancer Institute of Iran, Tehran University of Medical Sciences, Tehran, Iran

* Corresponding author. E-mail address: mona@alimohammadi.co.uk Author’s E-mails: m.amin.abazari@gmail.com, denizrafiei77@gmail.com, msoltani@uwaterloo.ca 


\begin{abstract}
Type-B aortic dissection (AD) is one of the greatest complex and fatal conditions with co-occurring disorders, challenging to treat. The initial treatment for patients presenting with $\mathrm{AD}$ is medical intervention to stabilize the condition. In the present study, a patient-specific geometry of type-B AD is generated from computed tomography images, and a three-element Windkessel lumped parameter model is implemented at the outlets to realistic boundary conditions. According to the physiological response of the antihypertensive drugs in the reduction of aortic blood flow and heart rate, three case studies with different heart rates have been created. Hemodynamic distributions including wall shear stress indicators, velocity and pressure are investigated and compared in each model. Results show that there is a considerable reduction in pressure furthermore, time-averaged wall shear stress (TAWSS) values decreased by $25 \%$ and $30 \%$, respectively. Main goal is to critically analysis the use of biomechanical and computational simulation tools to measure hemodynamic parameters in the absence and presence of antihypertensive drugs. It would be of significant use to clinicians to improve diagnostic and treatment planning.
\end{abstract}

\title{
Keywords:
}

Type-B aortic dissection, Patient-specific, Computational fluid dynamic (CFD), Windkessel model, Anti impulse therapy

\section{Introduction}

Cardiovascular disease (CVD) is the first most common cause of death worldwide $[1,2]$. Only $35 \%$ of patients survive after treatment [3]. Aortic dissection (AD) occurs when an injury to the intima layer enables blood to enter the middle layer of the aortic wall and causing the formation of false lumen (FL) as opposed to the original pathway; true lumen (TL), separated by the intimal flap (IF). This can occur in either the ascending or descending aorta, for which the disease is classified as Stanford type-A or type-B AD, respectively [4-6]. Rupture of the vessel wall, hypertension, malperfusion and aneurysm formation may be due to $\mathrm{AD}[7-11]$.

For cases of type-A $\mathrm{AD}$, prompt surgical intervention is generally required, while the ideal decision of treatment for type-B AD stays unknown and controversial. The treatment of type-B AD is more changeable and patient-specific [4,6,11-13] early intervention for type- $\mathrm{B} A \mathrm{D}$ patients is pharmacological therapy, to control manifestations by stabilising and decreasing the heart rate and blood pressure (BP) 
$[4,6,14]$. However, underlying and high-risk conditions such as malperfusion, aneurysm formation and aortic expansion may prioritize surgical treatment over pharmacological therapy; this decision is made by balancing the advantages of a successful surgery against confounding risk on a patient-to-patient basis $[4,15]$.

A successful planning treatment could potentially minimise the complication risk and obviate aortic reoperation [3]; the rate of reoperation for patients treated with surgery was about $10 \%$ higher than for patients treated medically $[3,15]$. In general, the rates of freedom from late aortic complications or late death have been similar for patients who treated medically and surgery, but for the last 30 years, the consensus has been showing that type-B AD patients should be treated medically except in the presence of life-threatening complications such as hypertension $[3,11,12]$. The two main reasons for the progression of the $\mathrm{AD}$ are increased blood flow and elevated BP $[12,15]$. In acute AD patients, the primary purposes of pharmacological treatment are to stabilise the dissection, accelerate healing, prevent rupture and reduce the risk of complications [12].

Pharmacological therapy has been used prior to surgical treatment or endovascular treatment $[12,16]$. Historically, in the early $1960 \mathrm{~s}$, according to the results of Wheat and associates experiments, it was found that two principal goals of pharmacological therapy are diminution of left ventricular ejection force $(\mathrm{dp} / \mathrm{dt})$ and reduction in BP $[12,16]$. A standard group of drugs such as an $\alpha$-adrenergic and $\beta$-adrenergic antagonist and Labetalol, in combination with beta-blockers (BBs) and sodium nitroprusside, are prescribed for routine medical therapy of type-B AD [12]. Trimethaphan and BB family groups would provide to decrease both the systemic BP and aortic pulse (dp/dt) and [12,17-19]. Although, BBs have a higher risk of stroke, death, and severe cardiovascular events than other antihypertensive drugs [18]. BBs are the initial pharmacological therapy for controlling the heart rate, aortic wall stresses, and BP; systolic BP should be lower than $120 \mathrm{mmHg}$ and heart rate lower than 60 BPM by taking BBs and also for additional BP reduction intravenous therapy must be used [11,20,21].

The physiological response of BBs reduces the progression and control of the type$\mathrm{B} A \mathrm{AD}$ by affecting the levels of potassium and calcium ions by increasing the effective refractory period (ERP) and cellular action potential duration (APD) $[22,23]$. Causing the reduction of aortic blood flow and reducing the heart rate [12]. Cardiac tamponade, aortic hemorrhage and myocardial ischemia can be the results of hypotension and shock [7,11]. One of the most crucial hemodynamic parameters in patients treated medically is the wall shear stress (WSS), which is 
shown in equation (1), where $\mu$ is the local dynamic viscosity and $\vec{u}$ is the fluid velocity vector:

$$
\tau=\mu \frac{\partial \vec{u}}{\partial y}
$$

The WSS can lead to dissection progression (FL progression), redissection, forming plaque in arteries, or aneurysm formation and reoperation; therefore, BBs and other related drugs are prescribed to minimise elevated WSS and prevent surgical or endovascular repair $[4,5,11,20,21,24]$. Therefore, by having a meaningful understanding of WSS data and other WSS indices, i.e., oscillatory shear index (OSI) and highly oscillatory, low magnitude shear (HOLMES) [25] in the absence and presence of BBs can be used as a blueprint for clinicians in their decision-making process. Typical co-occurring conditions in type-B AD patients are atherosclerosis $[11,25]$. Vascular calcification is a result of the hardening of the aorta and is related to hypertension and atherosclerosis [25]. The results of follow-up mortality in acute type B AD Patients were shown that the history of atherosclerosis causes a significant increase in the mortality rate [26]. Therefore, identifying the location of plaque non-invasive and using the shear stress index [25] can be of great help to the clinicians.

In this paper, a numerical simulation to investigate the hemodynamic parameters resulting from taking the $\mathrm{BB}$ family groups in an individual patient with type $\mathrm{B}$ $\mathrm{AD}$ is produced.

\section{Material and methods}

\section{Geometry and grid generation}

The three-dimensional (3D) fluid domain was generated from a stack of 887 digital imaging and communications in medicine (DICOM) images of a 58year-old male patient with Stanford type-B AD. The fluid domain was reconstructed using MIMICS Research 21.0 (2018 release, Materialise, Leuven, Belgium). The resolution of the CT images was $0.5 \mathrm{~mm} / \mathrm{pixel}$, and the IF was between 1.5-2.5 $\mathrm{mm}$ thick between the two tears. Different semiautomatic threshold, flood fill, and multiple slice edit tools have been used to create different masks to fully capture the geometry, retaining three-branch arteries (i.e. subclavian artery, left carotid artery, and brachiocephalic artery) on the aortic arch, and excluding other small vascular branches, due to the low quality of the images. The iliac arteries were omitted, and finally, boundaries cut with parallel planes in addition to inlet and outlets getting cut flat along the same axis. As shown in Fig.1, the cross-sectional 
contours (planes A and B) of the reconstructed 3D geometry are mapped back to computed tomography scans to better understand the 3D model representing the patient-specific vessel lumen.

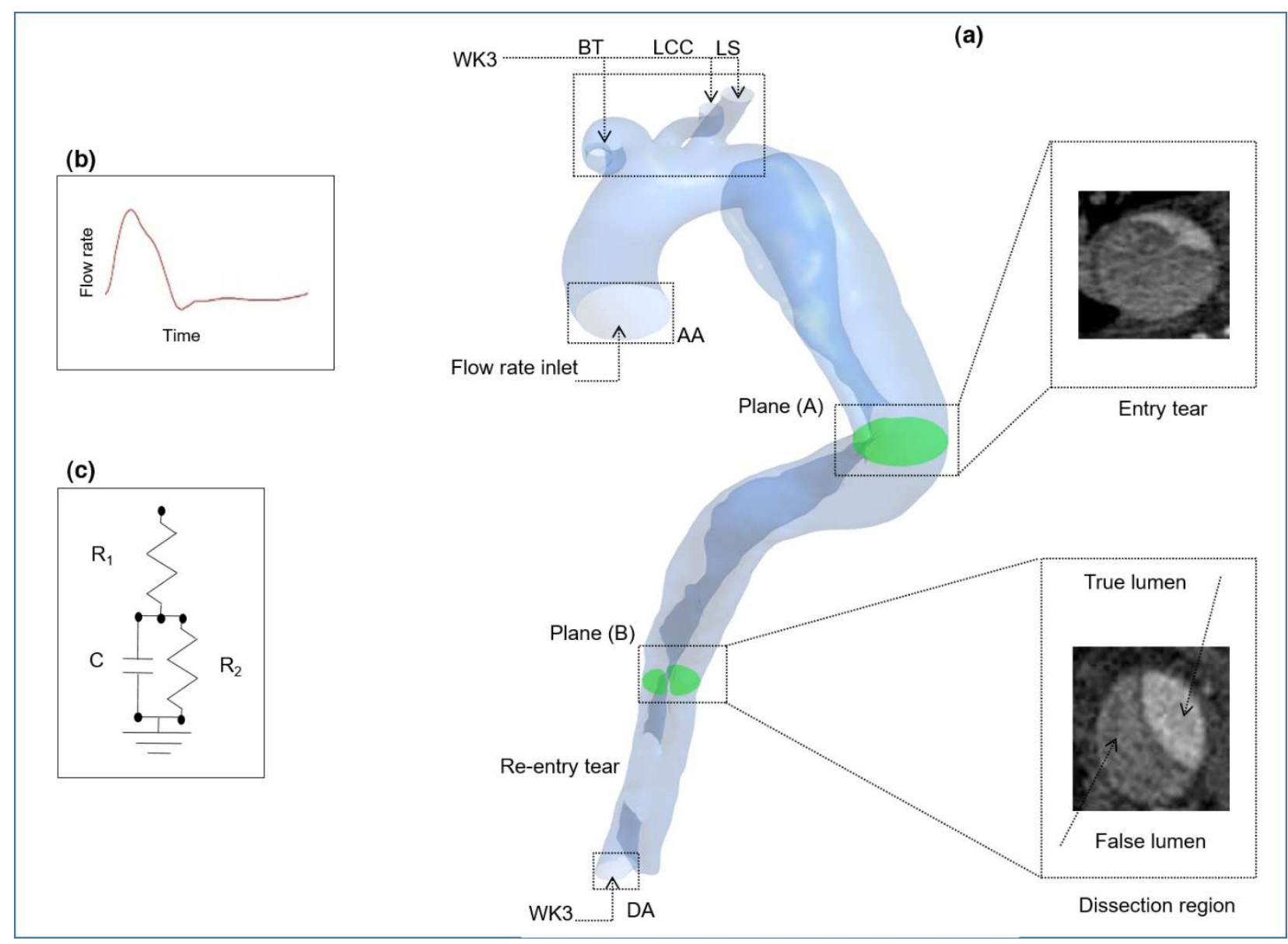

Figure 1. Geometry and boundary conditions. (a) Patient-specific dissected aorta geometry with two planes to illustrate the dissection region and entry tear. The boundaries are names with black arrows: DA: descending aorta, LS: left subclavian artery, AA: ascending aorta, LCC: left common carotid artery, BT: brachiocephalic trunk (b) Flow rate inlet diagram; (c) 3-element Windkessel boundary condition.

The fluid domain was meshed using ANSYS Meshing 18.2 (ANSYS Inc., Canonsburg, PA, USA). The geometry consisted of about 420000 and 175000 tetrahedral cells and node numbers, respectively. To minimise the computational errors near the wall, seven prismatic layers with a growth rate of 1.2 are deployed. Mesh independence has been done for the fluid domain, a coarse mesh with about 250,000 cells, and a fine-mesh with about 1000000 cells were generated to evaluate the grid independency. The pressure values in these three cases were examined so that there was a maximum 3.5\% difference between the coarse mesh 
relative to the medium mesh and a $0.7 \%$ maximum difference between the medium mesh and the fine mesh. In order to save computer costs, the medium mesh is chosen.

\section{Computational fluid dynamics}

The blood was assumed as an incompressible fluid with a density [4] of 1056 $\mathrm{kg} / \mathrm{m}^{3}$. The fluid was considered as a non-Newtonian model, with viscosity determined by Carreau-Yasuda viscosity model wherein $\mu$ is viscosity, $\dot{\gamma}$ is shear rate, $\mu_{0}$ is Carreau Yasuda zero shear viscosity and $\mu_{\infty}, a, m$ and $\lambda_{C Y}$ are CarreauYasuda infinite shear viscosity, Yasuda exponent, Carreau-Yasuda Power Law Index and Carreau-Yasuda time constant, respectively:

$$
\mu=\left(\mu_{0}-\mu_{\infty}\right)\left(1+\left(\lambda_{C Y} \dot{\gamma}\right)^{a}\right)^{(m-1) / a}+\mu_{\infty}
$$

The parameters used in the present study were determined by Gijsen et al. [27] that can be seen in table (1).

Table 1. Material properties used for the Carreau-Yasuda model [27].

\begin{tabular}{ccccc}
\hline$\mu_{0}(\mathrm{~m}$ pa s $)$ & $\mu_{\infty}(\mathrm{m}$ pa s $)$ & $a$ & $m$ & $\lambda_{C Y}(\mathrm{~s})$ \\
\hline 22 & 2.2 & 0.644 & 0.392 & 0.110 \\
\hline
\end{tabular}

The continuity and Navier-Stokes equations (equations (3) and (4), respectively) were solved using by the computational fluid dynamics (CFD) software ANSYSCFX 18.2 (ANSYS Inc., Canonsburg, PA, USA) based on the finite volume method, discretization of the equations involved a second-order backward Euler scheme with a time step of $0.005 \mathrm{~s}$, and the maximum residual mean square errors were set to $10 \mathrm{e}-5$ :

$$
\begin{gathered}
\nabla \cdot \vec{u}=0 \\
\rho \frac{\partial \overrightarrow{\mathrm{u}}}{\partial \mathrm{t}}+\rho(\overrightarrow{\mathrm{u}} \cdot \nabla) \overrightarrow{\mathrm{u}}+\nabla p-\mu \Delta \overrightarrow{\mathrm{u}}=0
\end{gathered}
$$

where $\rho$ and $p$ represent the density and pressure, respectively. The blood flow was assumed to be laminar [4,28,29]. Reynolds number in the AA was approximately 1900 . This usually occurs in large arteries due to the low mean flow velocity $[4,30]$. In order to mimic patients undergoing pharmacological treatment, the heart rate must be reduced (below $60 \mathrm{BPM}$ ) [11,20,21]. For this purpose, three case studies with type-B AD with different heart rates have been created to 
investigate. As a result of taking BBs, the patient's heart rate drops from 86 BPM (high heart rate) to $70 \mathrm{BPM}$ (moderate heart rate) and finally to $55 \mathrm{BPM}$ (regular heart rate). The input flow profile is virtually adjusted to represent the three aforementioned heart rates (rate reduced using beta-blockade). The input flow rate diagrams in all three cases are shown in Fig. 2.

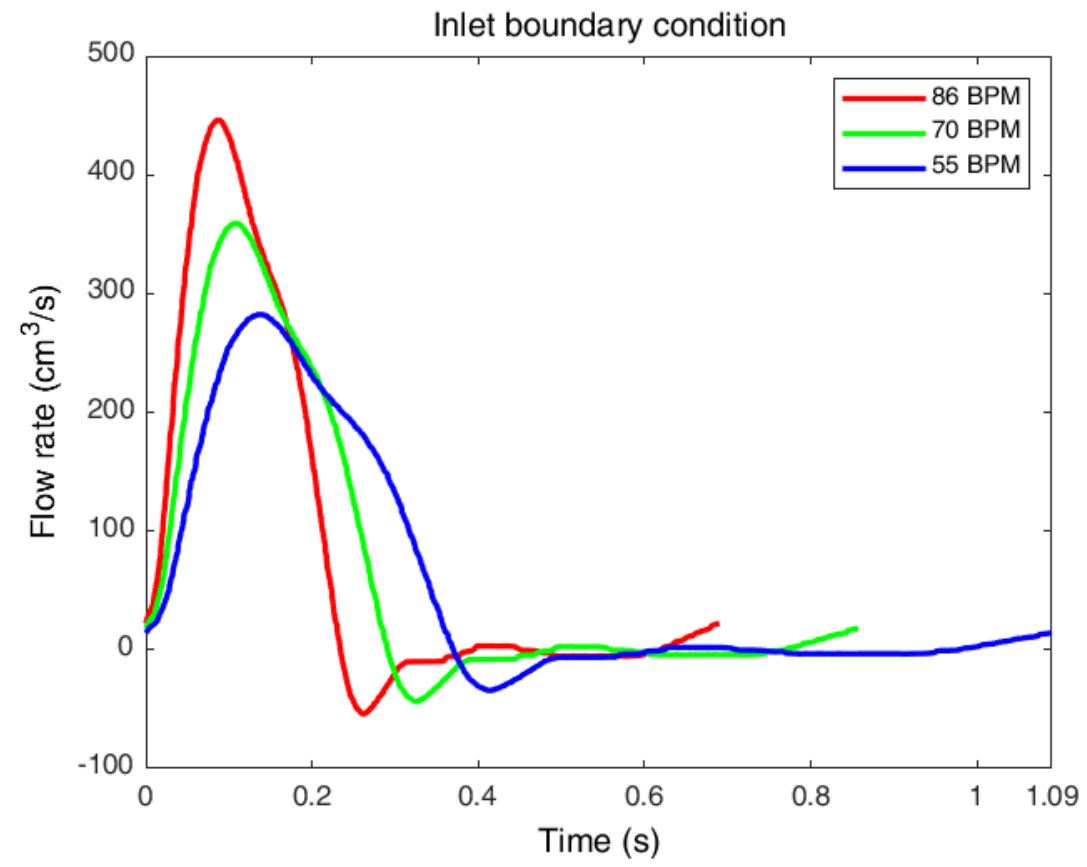

Figure 2. Input flow rate diagrams at the ascending aorta for all three heartbeats.

\section{Boundary conditions}

The inlet flow waveform to the AA was not available for this patient, so Karmonik et al.'s results [31] have been used and adjusted accordingly. Three cardiac cycles were needed to reach periodic repetitive states, and the last cycle was used to extract all the results. ANSYS CFD-Post and MATLAB (R2018b version, Mathworks, Natick, USA) were used to Post-processing the data. As discussed in Alimohammadi et al.'s papers [7,32], the present study was carried out in order to provide a framework for clinicians to better understand and tune their patientspecific pharmacological treatment. Therefore, computational cost (simulation time) is of great importance herein; on the other hand, the inclusion of vessel wall simulation (Fluid-Solid Interaction) would require great resources and adequate time [7,33,34]; thus, rigid walls are assumed, and the aorta was assumed as no-slip walls. A three-element Windkessel lumped parameter model is implemented at the outlets to realistic boundary conditions. The values of the model parameters are also taken from a similar study (Fig. 1c) [25]. 


\section{Results}

\section{Velocity distribution}

The velocity distribution through all cases is shown in Fig. 3. Generally, the velocity values decreased with reducing heart rate in all cardiac points except pick systole, where no significant changes can be seen. The velocity through the FL decreased, which lead to reducing the velocity gradient in the WSS equation (see equation (1)).

Figure 4 demonstrates streamlines at end-diastole in three cases. Also, the distribution of volume renderings of the velocity magnitude on a plane in the middle of the entry tear is shown. The maximum velocity is seen in the model with $86 \mathrm{BPM}$ at entry tear. More specifically, in the case of 86 BPM heart rate, the maximum velocity of flow jet was about $1 \mathrm{~m} / \mathrm{s}$ around the entry tear, which reduced to about $0.7 \mathrm{~m} / \mathrm{s}$ and $0.47 \mathrm{~m} / \mathrm{s}$ at 70 and $55 \mathrm{BPM}$, respectively. On the other hand, by lowering the heartbeats, the velocity values and turbulence regions at entry tear were decreased.
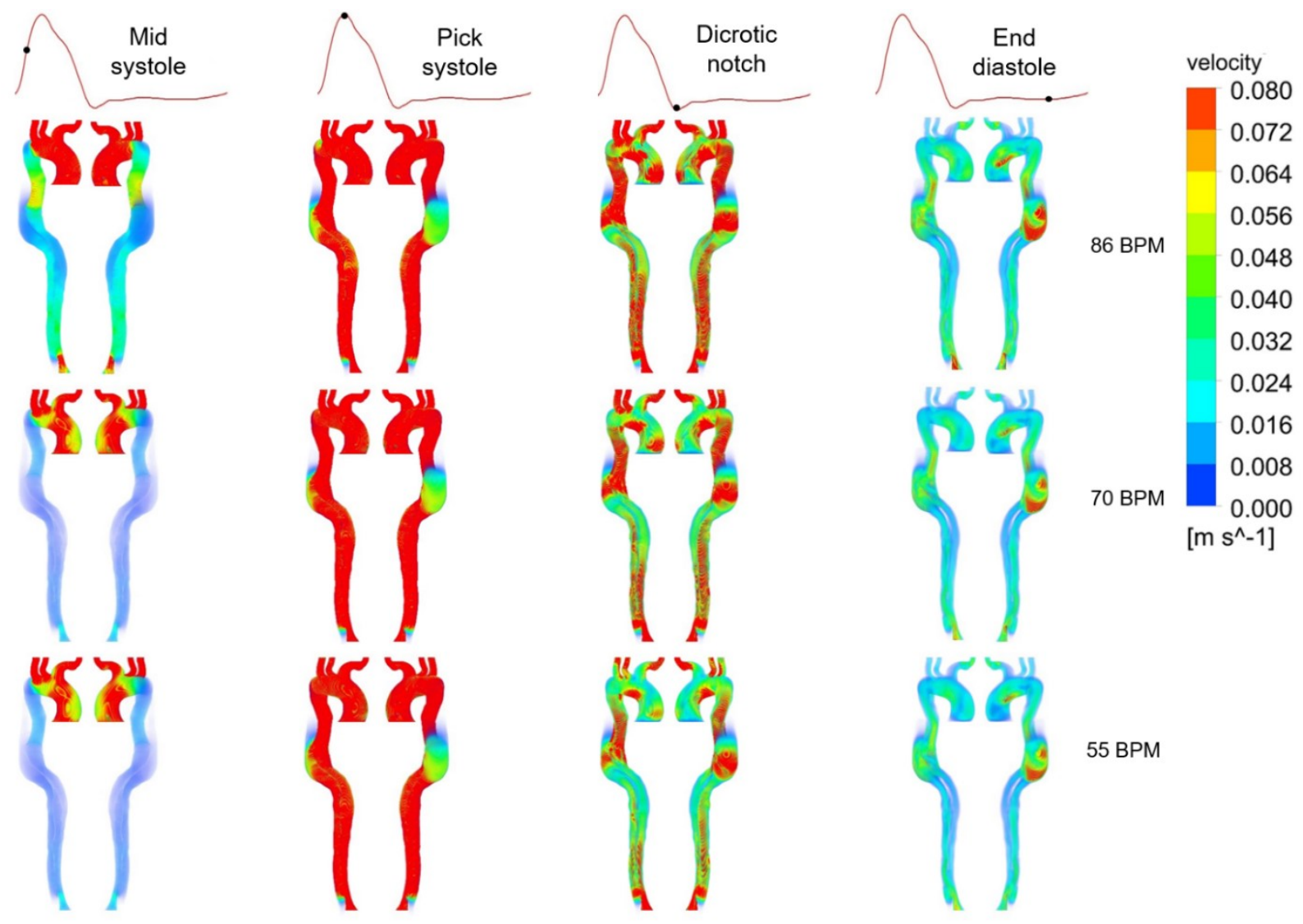

Figure 3. Velocity distribution for all three cases at four cardiac points. 


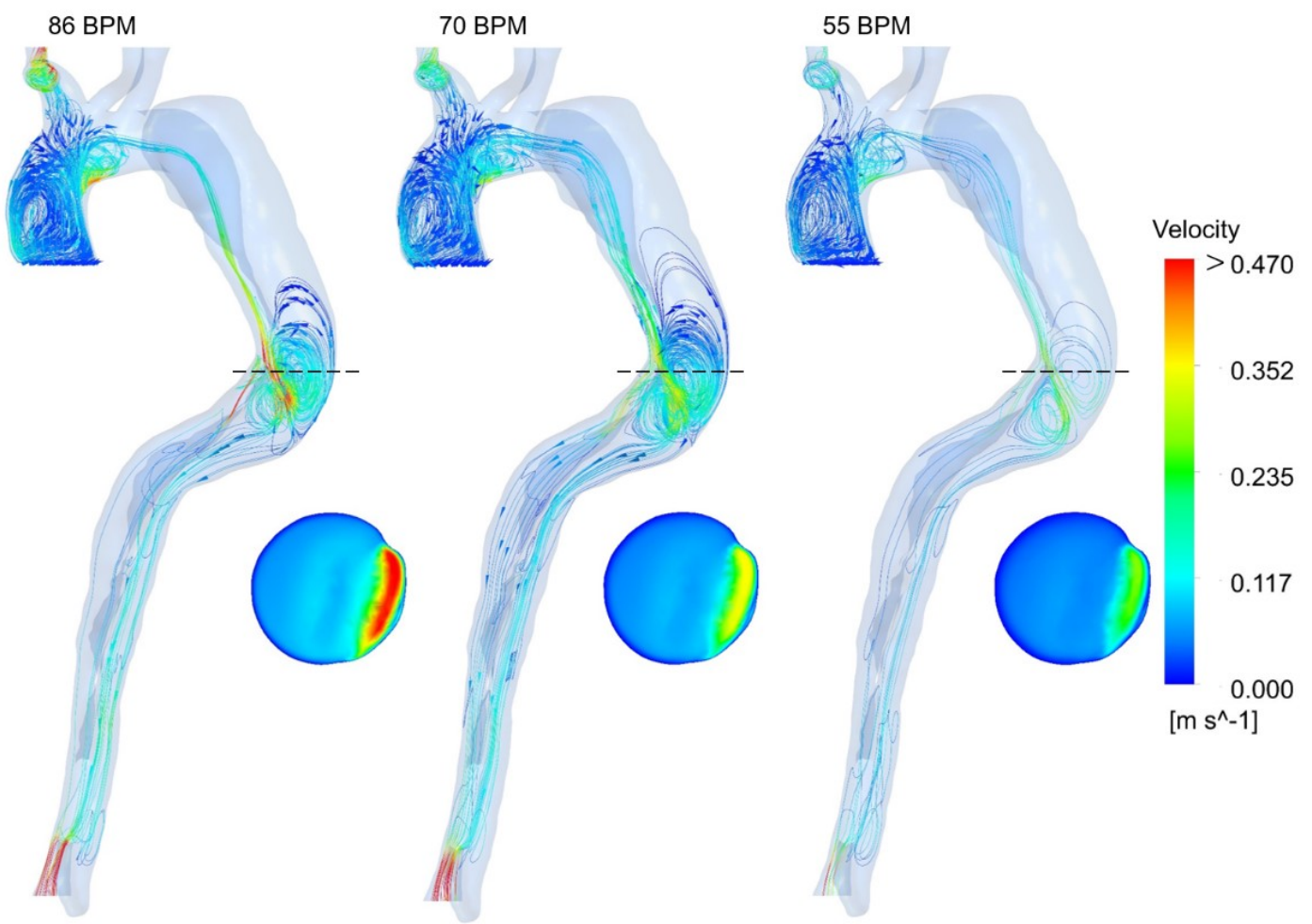

Figure 4. Velocity magnitude and streamlines for the three models at end-diastole.

\section{Pressure distribution}

The pressure distribution through all cases at pick systolic BP is shown in Fig. 5. Results show a significant difference in pressure values between the different heartbeats. The average pressure was decreased from about $110 \mathrm{mmHg}$ at $86 \mathrm{BPM}$ to approximately 100 and $85 \mathrm{mmHg}$ at 70 and $55 \mathrm{BPM}$, respectively. 


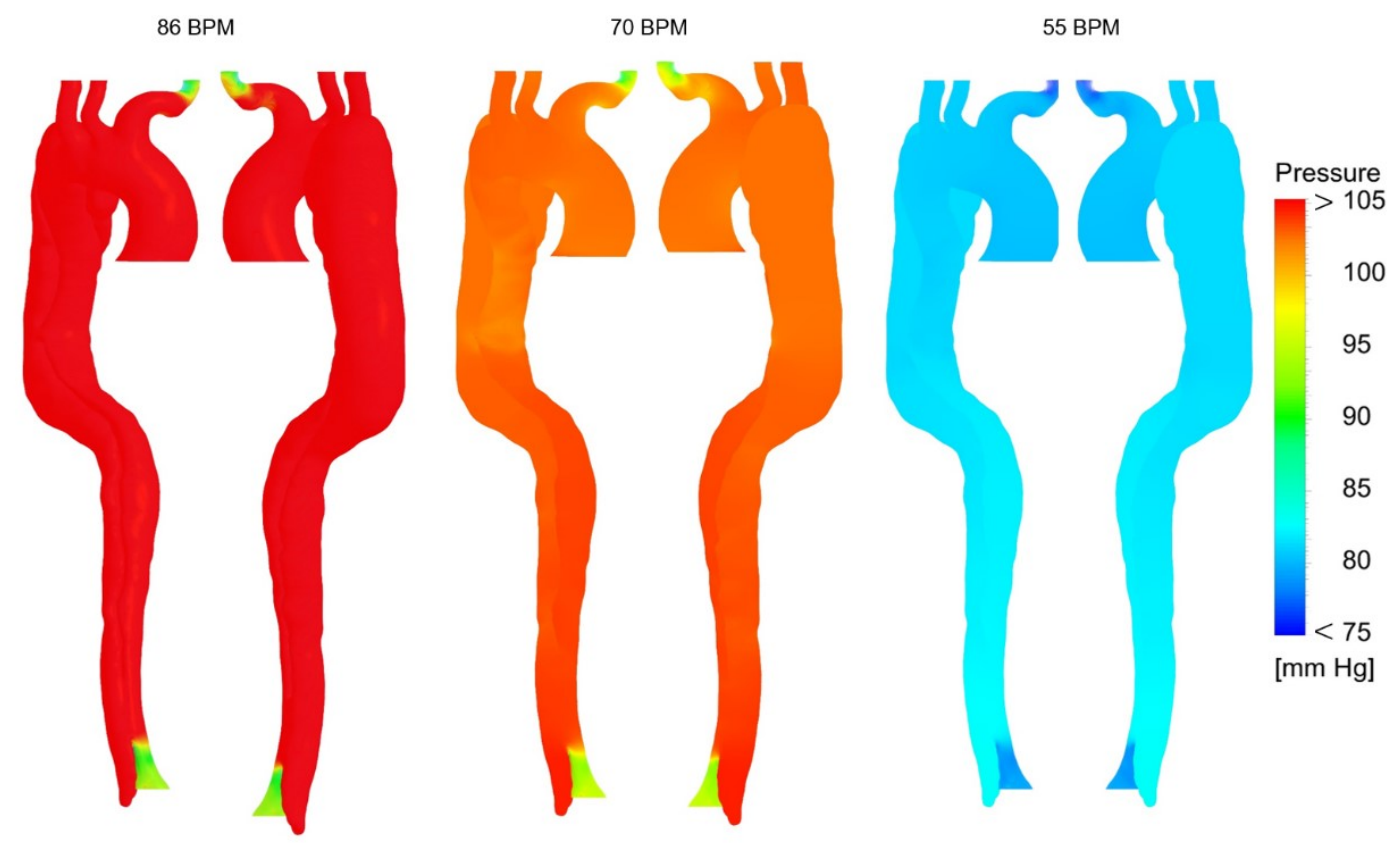

Figure 5. Pressure distribution for three cases at pick systolic blood pressure.

\section{WSS indicators}

Time-averaged wall shear stress (TAWSS) is an important WSS indicator which is prescribed by equation (5) [4]:

$$
T A W S S=\frac{1}{T} \int_{0}^{T}|\vec{\tau}(t)| d t
$$

Where $\vec{\tau}(t)$ is the WSS vector at time $t$. This equation is adjusted to all $3 \mathrm{D}$ geometry nodes to evaluate TAWSS. The TAWSS distribution and their percentage differences are shown in Fig. 6 and Fig. 7, respectively.

As shown in Fig. 6, the highest values of TAWSS were seen in the upper branches (especially BT) and around DA. The lowest TAWSS is seen in downstream FL, due to the reduction of velocity gradient, according to equations (1) and (5). From Fig. 7, it can be seen that TAWSS values in the whole of the aorta were decreased except for the FL after aortic arch (proximal FL) and around the DA in 86 BPM case. However, according to Fig. 6 , in positive terms (about $0.2 \mathrm{~Pa}$ ), it is apparent that TAWSS has been lower in these areas, and with increasing the percentage difference between 86 and $70 \mathrm{BPM}$, its value has not changed significantly. Figure 7 illustrates TAWSS is decreased by an average of $25 \%$ and $30 \%$ at entry tear in each case, respectively. And it can be easily understood that TAWSS decreased by about $25 \%$ through the FL. 


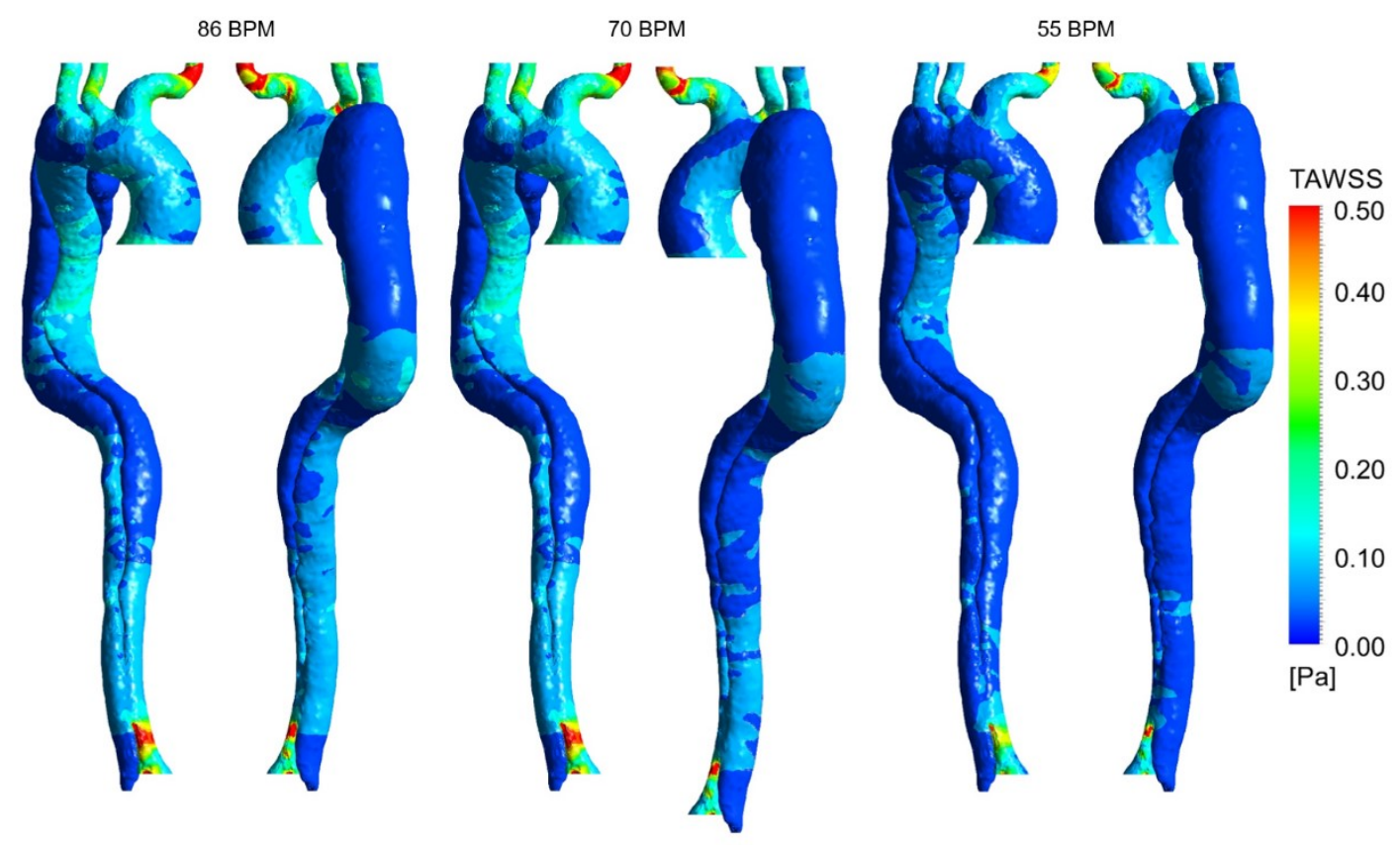

Figure 6. Time-averaged wall shear stress (TAWSS) distribution for three cases.

(a)

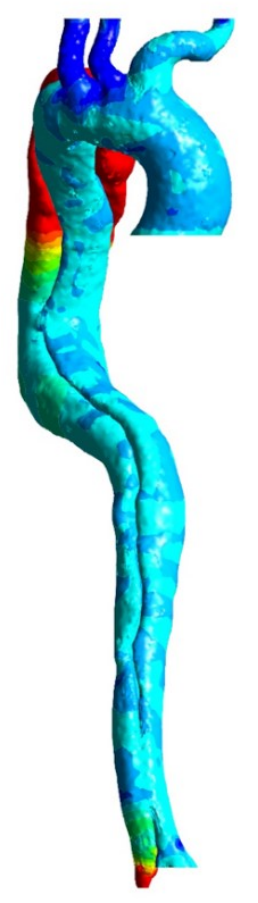

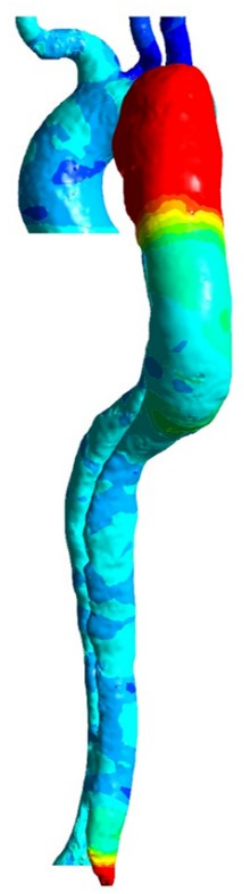

(b)
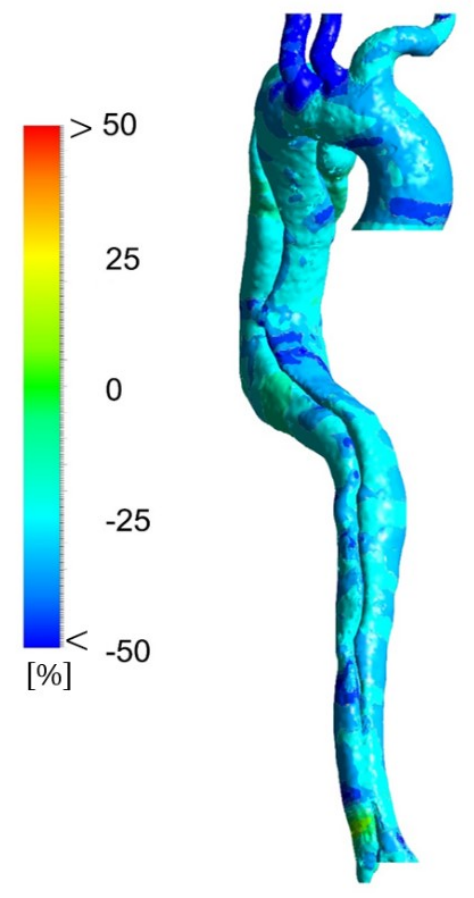

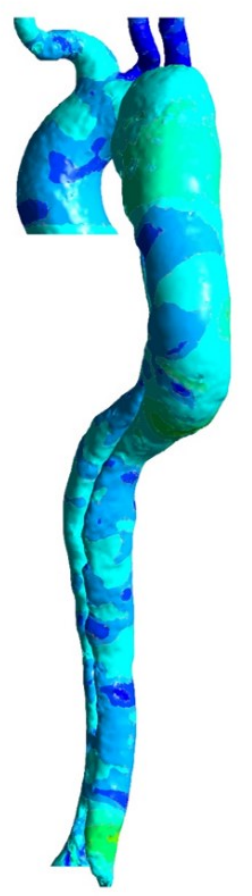

Figure 7. Percentage difference in time-averaged wall shear stress (TAWSS) relative to different heart rates. (a) Percentage difference in TAWSS between 86 BPM and 70 BPM; (b) Percentage difference in TAWSS between 55 BPM and 70 BPM. 
OSI is another meaningful WSS indicator and can be achieved from the following equation [4]:

$$
O S I=\frac{1}{2}\left(1-\frac{\left|\frac{1}{T} \int_{0}^{T} \vec{\tau}(t) d t\right|}{T A W S S}\right)
$$

This parameter measures the oscillation of forces on the endothelial cells [4] and has a value between 0 and 0.5 , a zero OSI quantity coincides with one-way wall shear forces, and the higher values indicate that the direction of the WSS forces is rather unknown $[4,5]$.

The OSI distribution and their percentage differences are shown in Fig. 8 and Fig. 9, respectively. Generally, as shown in Fig. 8, high OSI can be seen in the dispersed elevated regions in the downstream entry tear and around the DA due to the complicated and unstable flow.

According to Fig. 9, OSI has not changed significantly throughout the aorta. In fact, the average percentage difference in OSI was approximately equal to $\pm 10 \%$ in most parts. Also, over the TL, a contrast of around $-18 \%$ can be seen, which indicated a decrease in the oscillating nature of the WSS forces. According to Fig. 9a, the highest percentage difference reduction in OSI was in the FL after aortic arch and DA.
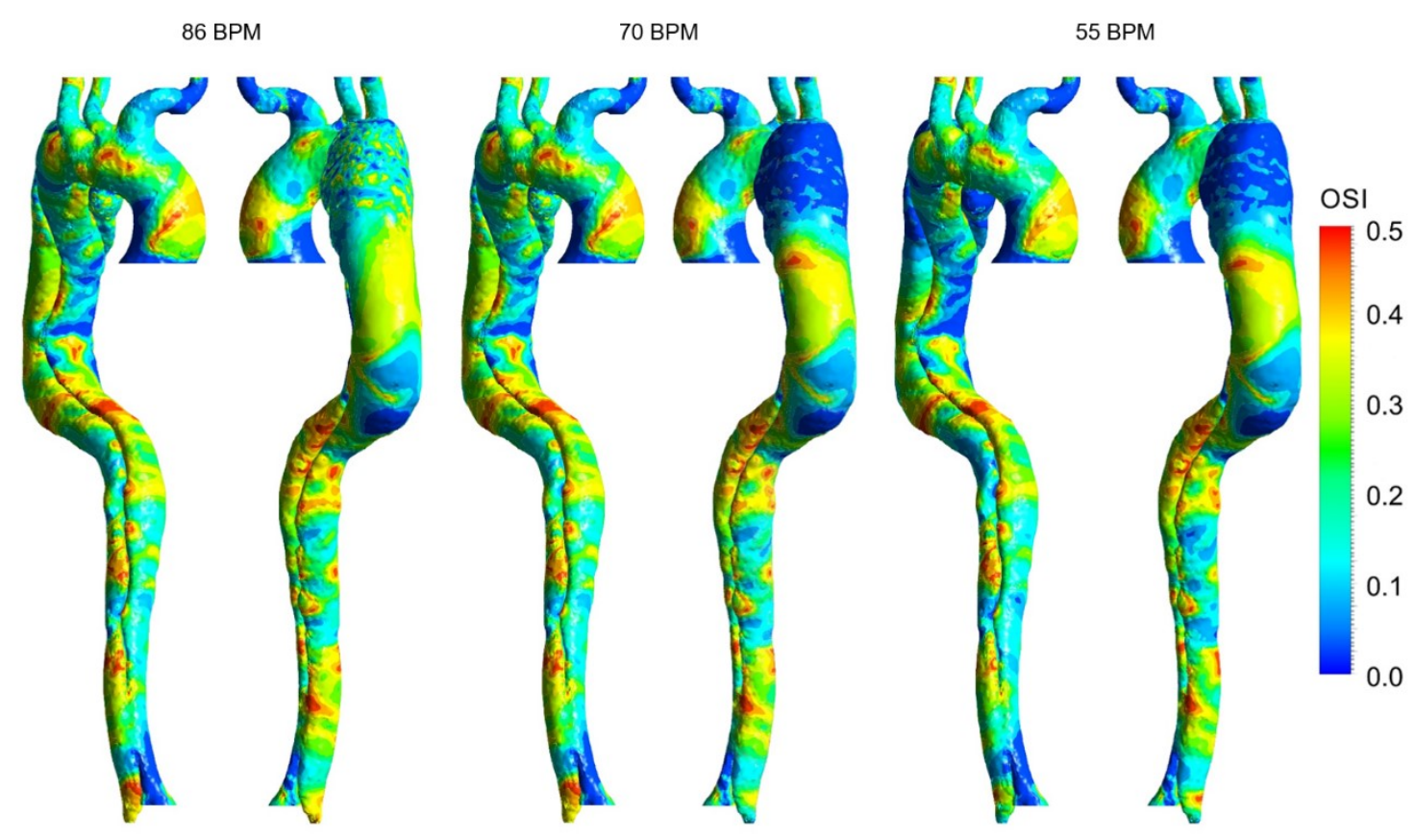

Figure 8. Oscillatory shear index (OSI) distribution for three cases. 
(a)

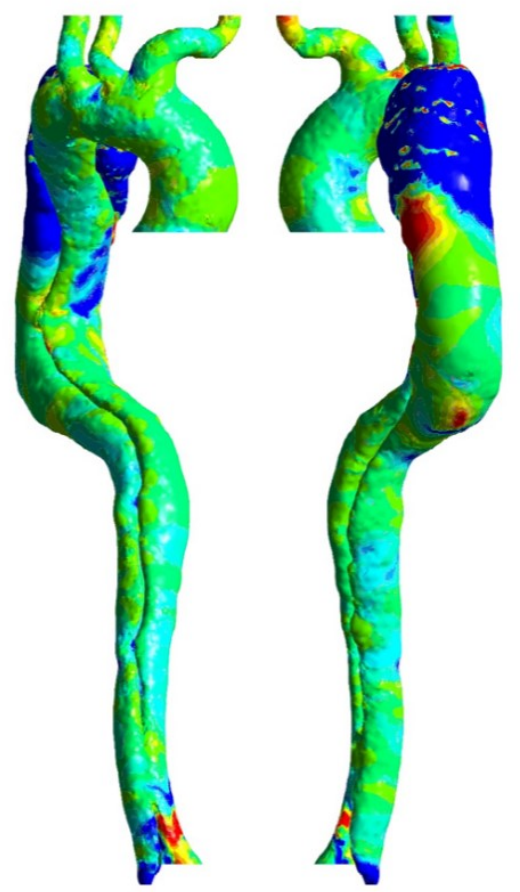

(b)

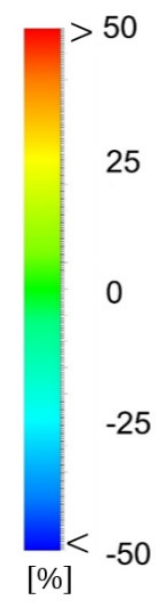

Figure 9. Percentage difference in oscillatory shear index (OSI) relative to different heart rates. (a) Percentage difference in OSI between 86 BPM and 70 BPM; (b) Percentage difference in OSI between 55 BPM and 70 BPM.

For better understanding of the combination and interaction of these two characteristics (TAWSS and OSI), a parameter called HOLMES is investigated, given by [25]:

$$
\text { HOLMES }=\text { TAWSS }(0.5-O S I)
$$

HOLMES is a key parameter for predicting the location of plaques and their progression [11]. HOLMES distribution and their percentage differences are shown in Fig. 10 and Fig. 11, respectively. According to Fig.10, the mean value of HOLMES was less than $0.5 \mathrm{~Pa}$ throughout the domain except for the upper branches (especially BT) and around the DA.

From Fig. 11, it can be noted that HOLMES has decreased significantly through the aorta except in few regions, i.e., FL after the aortic arch (proximal FL) and DA (however, without any critical changes in absolute term). By decreasing each stage of heartbeats, HOLMES at the TL and FL was decreased by an average of about $25 \%$ and $22 \%$, respectively. 

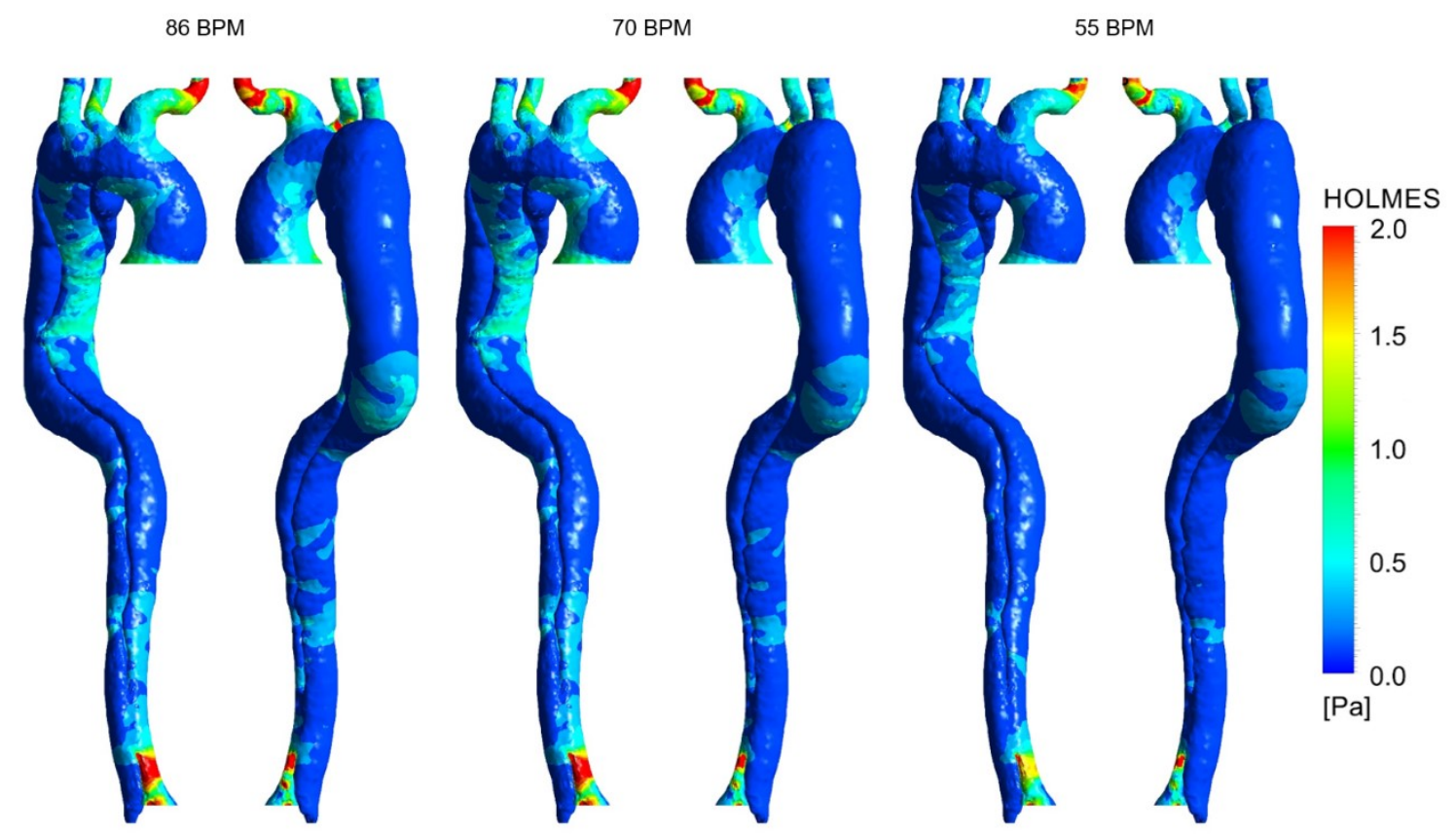

Figure 10. Highly oscillatory, low magnitude Shear (HOLMES) distribution for three cases.

(a)

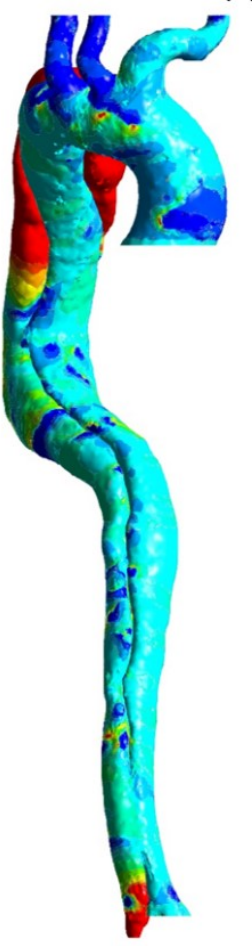

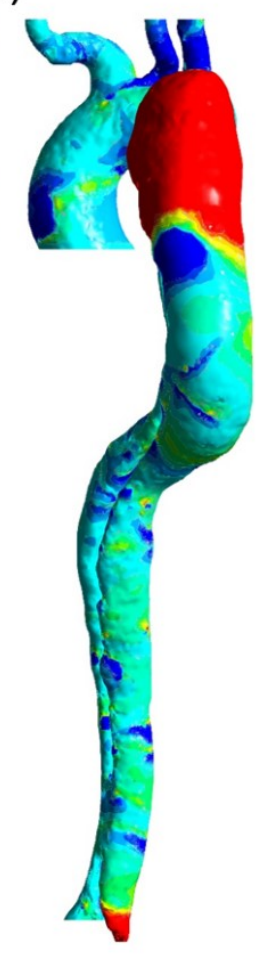

(b)
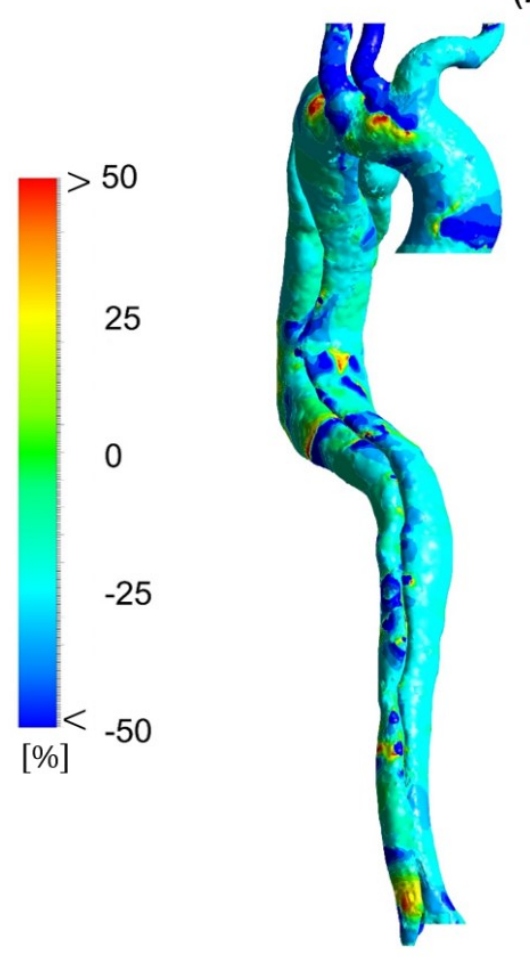

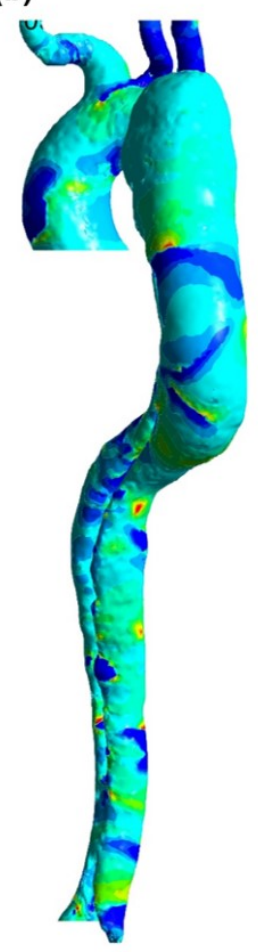

Figure 11. Percentage difference in highly oscillatory, low magnitude Shear (HOLMES) relative to different heart rates. (a) Percentage difference in HOLMES between 86 BPM and 70 BPM; (b) Percentage difference in HOLMES between 55 BPM and 70 BPM. 


\section{Discussion}

In the present work, the results showed significant hemodynamic differences between the three cases. In models with 70 and 55 BPM, the flow jet in the entry tear at end-diastole has decreased by about $42 \%$ and $48 \%$ in each stage of lowering heart rate, respectively and the vortical structures around the entry tear were also reduced. The vortical structures cause platelets to be trapped, and in the downstream regions, decreasing WSS causes thrombosis $[35,36]$.

In $71 \%$ of patients with type-B AD, systolic BP was more than $150 \mathrm{mmHg}$ [11]. Studies have shown that calcification, extracellular fatty acid deposition, wall thickening and fibrosis are driven by chronic exposure to high pressure [5]. When comparing pressure values, using the results shown in Fig. 5, the pressure gradient along the whole aorta is significantly decreased; this behaviour indicates the successful effect of antihypertensive drugs [12,16,30]

The WSS is principally essential in the study of AD because of its direct effect on the vessel wall $[4,5]$, which cannot be measured invasively or experimentally. However, CFD can provide a great insight into this parameter and its resultant indices $[4,5,29]$. For example, a high TAWSS value causes the FL to expand and grow, increasing the risk of rupture in this area $[4,25]$ and closing the TL and total loss of function of the lower appendages $[4,37]$.

Due to the decreasing heart rate, the velocity gradient through the coarctations was decreased and led to reduce WSS. As expected, by approximately a $25 \%$ reduction in TAWSS values at the FL, this area's growth and expansion have been prevented. As reported by the previous research $[25,38]$, if an area has a high OSI and a low TAWSS, there is a high risk of rupture, calcification, or wall thickening there. The current results showed, although decreasing TAWSS in coarctations, OSI becomes more uniform where no considerable changes in its absolute terms can be seen. So that hypothesis prevents by lowering in heart rate. By notable decreasing in HOLMES in the TL and FL thus reducing the risk of endothelial cell permeability and plaque formation with successful virtual medication [25]

This study represents a prime example of using computer simulations to measure hemodynamic parameters as a diagnostic tool for the clinicians. To investigate in detail, the predictive power of the model, the next steps will include fluid-solid interaction studies of different patients to allow comparisons between patients and possibly invasive analyses. 


\section{Data Availability}

The datasets used and analysed during the current study are available from the corresponding author on reasonable request.

\section{References}

1 Yacoub, M., ElGuindy, A., Afifi, A., Yacoub, L. \& Wright, G. Taking cardiac surgery to the people. Journal of cardiovascular translational research 7, 797-802 (2014).

2 Zhang, C.-L., Long, T.-Y., Bi, S.-S., Sheikh, S.-A. \& Li, F. CircPAN3 ameliorates myocardial ischaemia/reperfusion injury by targeting miR-421/Pink1 axis-mediated autophagy suppression. Laboratory Investigation, doi:10.1038/s41374-020-00483-4 (2020).

3 Umaña, J. P., Miller, D. C. \& Mitchell, R. S. What is the best treatment for patients with acute type B aortic dissections-medical, surgical, or endovascular stent-grafting? The Annals of thoracic surgery 74, S1840-S1843 (2002).

4 Alimohammadi, M. Aortic dissection: simulation tools for disease management and understanding. (Springer, 2018).

5 Peng, L. et al. Patient-specific Computational Hemodynamic Analysis for Interrupted Aortic Arch in an Adult: Implications for Aortic Dissection Initiation. Scientific Reports 9, 8600, doi:10.1038/s41598-019-45097-z (2019).

6 Nienaber, C. A. et al. Aortic dissection. Nature Reviews Disease Primers 2, 16053, doi:10.1038/nrdp.2016.53 (2016).

7 Alimohammadi, M. et al. Evaluation of the hemodynamic effectiveness of aortic dissection treatments via virtual stenting. The International journal of artificial organs $\mathbf{0}, 0$, doi:10.5301/ijao.5000310 (2014).

8 Criado, F. J. Aortic dissection: a 250-year perspective. Texas Heart Institute journal 38, 694-700 (2011).

9 Fattori, R. et al. Complicated acute type B dissection: is surgery still the best option?: a report from the International Registry of Acute Aortic Dissection. JACC. Cardiovascular interventions 1, 395-402, doi:10.1016/j.jcin.2008.04.009 (2008).

10 Hagan, P. G. et al. The International Registry of Acute Aortic Dissection (IRAD): new insights into an old disease. Jama 283, 897-903, doi:10.1001/jama.283.7.897 (2000).

11 Hiratzka, L. F. et al. 2010 ACCF/AHA/AATS/ACR/ASA/SCA/SCAI/SIR/STS/SVM guidelines for the diagnosis and management of patients with Thoracic Aortic Disease: a report of the American College of Cardiology Foundation/American Heart Association Task Force on Practice Guidelines, American Association for Thoracic Surgery, American College of Radiology, American Stroke Association, Society of Cardiovascular Anesthesiologists, Society for Cardiovascular Angiography and Interventions, Society of Interventional Radiology, Society of Thoracic Surgeons, and Society for Vascular Medicine. Circulation 121, e266-369, doi:10.1161/CIR.0b013e3181d4739e (2010).

12 Khan, I. A. \& Nair, C. K. Clinical, diagnostic, and management perspectives of aortic dissection. Chest 122, 311-328, doi:10.1378/chest.122.1.311 (2002).

13 Lortz, J. et al. High intimal flap mobility assessed by intravascular ultrasound is associated with better short-term results after TEVAR in chronic aortic dissection. Scientific Reports 9, 7267, doi:10.1038/s41598-019-43856-6 (2019).

14 Guidelines for diagnosis and treatment of aortic aneurysm and aortic dissection (JCS 2011): digest version. Circulation journal : official journal of the Japanese Circulation Society 77, 789-828, doi:10.1253/circj.cj-66-0057 (2013). 
15 Umaña, J. P. et al. Is medical therapy still the optimal treatment strategy for patients with acute type B aortic dissections? The Journal of thoracic and cardiovascular surgery 124, 896-910, doi:10.1067/mtc.2002.123131 (2002).

16 Gionis, M. N. et al. Medical management of acute type a aortic dissection in association with early open repair of acute limb ischemia may prevent aortic surgery. Am J Case Rep 14, 52-57, doi:10.12659/AJCR.883793 (2013).

17 Genoni, M. et al. Chronic beta-blocker therapy improves outcome and reduces treatment costs in chronic type B aortic dissection. European journal of cardio-thoracic surgery : official journal of the European Association for Cardio-thoracic Surgery 19, 606-610, doi:10.1016/s1010-7940(01)00662-5 (2001).

18 Pucci, G., Ranalli, M. G., Battista, F. \& Schillaci, G. Effects of $\beta$-Blockers With and Without Vasodilating Properties on Central Blood Pressure: Systematic Review and Meta-Analysis of Randomized Trials in Hypertension. Hypertension (Dallas, Tex. : 1979) 67, 316-324, doi:10.1161/hypertensionaha.115.06467 (2016).

19 Ziganshin, B. A., Dumfarth, J. \& Elefteriades, J. A. Natural history of Type B aortic dissection: ten tips. Ann Cardiothorac Surg 3, 247-254, doi:10.3978/j.issn.2225319X.2014.05.15 (2014).

20 Suzuki, T. et al. Medical management in type B aortic dissection. Ann Cardiothorac Surg 3, 413-417, doi:10.3978/j.issn.2225-319X.2014.07.01 (2014).

21 Tsai, T. T., Nienaber, C. A. \& Eagle, K. A. Acute aortic syndromes. Circulation 112, 38023813, doi:10.1161/circulationaha.105.534198 (2005).

22 Kharche, S. R. et al. Effects of human atrial ionic remodelling by $\beta$-blocker therapy on mechanisms of atrial fibrillation: a computer simulation. Europace 16, 1524-1533, doi:10.1093/europace/euu084 (2014).

23 Martinez-Pinna, J. et al. Oestrogen receptor $\beta$ mediates the actions of bisphenol-A on ion channel expression in mouse pancreatic beta cells. Diabetologia 62, 1667-1680, doi:10.1007/s00125-019-4925-y (2019).

24 Keshavarz-Motamed, Z. A diagnostic, monitoring, and predictive tool for patients with complex valvular, vascular and ventricular diseases. Scientific Reports 10, 6905, doi:10.1038/s41598-020-63728-8 (2020).

25 Alimohammadi, M., Pichardo-Almarza, C., Agu, O. \& Díaz-Zuccarini, V. Development of a Patient-Specific Multi-Scale Model to Understand Atherosclerosis and Calcification Locations: Comparison with In vivo Data in an Aortic Dissection. Front Physiol 7, 238-238, doi:10.3389/fphys.2016.00238 (2016).

26 Tsai, T. T. et al. Long-term survival in patients presenting with type A acute aortic dissection: insights from the International Registry of Acute Aortic Dissection (IRAD). Circulation 114, I350-356, doi:10.1161/circulationaha.105.000497 (2006).

27 Gijsen, F. J., van de Vosse, F. N. \& Janssen, J. D. The influence of the non-Newtonian properties of blood on the flow in large arteries: steady flow in a carotid bifurcation model. J Biomech 32, 601-608, doi:10.1016/s0021-9290(99)00015-9 (1999).

28 Andayesh, M., Shahidian, A. \& Ghassemi, M. Numerical investigation of renal artery hemodynamics based on the physiological response to renal artery stenosis. Biocybernetics and Biomedical Engineering 40, 1458-1468, doi:https://doi.org/10.1016/j.bbe.2020.08.006 (2020).

29 Bonfanti, M. \& Balabani, S. Computational tools for clinical support: a multi-scale compliant model for haemodynamic simulations in an aortic dissection based on multi-modal imaging data. 14, doi:10.1098/rsif.2017.0632 (2017).

30 Mohr-Kahaly, S. et al. Ambulatory follow-up of aortic dissection by transesophageal twodimensional and color-coded Doppler echocardiography. Circulation 80, 24-33, doi:10.1161/01.cir.80.1.24 (1989). 
31 Karmonik, C. et al. Preliminary findings in quantification of changes in septal motion during follow-up of type B aortic dissections. Journal of vascular surgery 55, 1419-1426, doi:10.1016/j.jvs.2011.10.127 (2012).

32 Bonfanti, M. et al. A simplified method to account for wall motion in patient-specific blood flow simulations of aortic dissection: Comparison with fluid-structure interaction. Medical engineering \& physics, doi:10.1016/j.medengphy.2018.04.014 (2018).

33 Rikhtegar Nezami, F., Athanasiou, L. S., Amrute, J. M. \& Edelman, E. R. Multilayer flow modulator enhances vital organ perfusion in patients with type B aortic dissection. $\mathrm{Am} J$ Physiol Heart Circ Physiol 315, H1182-H1193, doi:10.1152/ajpheart.00199.2018 (2018).

34 Keshavarz-Motamed, Z. et al. Effect of coarctation of the aorta and bicuspid aortic valve on flow dynamics and turbulence in the aorta using particle image velocimetry. Experiments in Fluids 55, 1696, doi:10.1007/s00348-014-1696-6 (2014).

35 Biasetti, J., Hussain, F. \& Gasser, T. C. Blood flow and coherent vortices in the normal and aneurysmatic aortas: a fluid dynamical approach to intra-luminal thrombus formation. Journal of the Royal Society, Interface 8, 1449-1461, doi:10.1098/rsif.2011.0041 (2011).

36 Menichini, C., Cheng, Z., Gibbs, R. G. \& Xu, X. Y. Predicting false lumen thrombosis in patient-specific models of aortic dissection. 13 (2016).

37 Bozinovski, J. \& Coselli, J. S. Outcomes and survival in surgical treatment of descending thoracic aorta with acute dissection. The Annals of thoracic surgery 85, 965-970; discussion 970-961, doi:10.1016/j.athoracsur.2007.11.013 (2008).

38 Qiao, Y. et al. Numerical simulation of two-phase non-Newtonian blood flow with fluidstructure interaction in aortic dissection. Computer methods in biomechanics and biomedical engineering 22, 620-630, doi:10.1080/10255842.2019.1577398 (2019).

\section{Author Contributions}

M. A. and M. A. A. conceived the idea. M. A. A. carried out the simulations, analyzed the data, and drafted the manuscript. D. R. participated in data analysis. M. S. and M. A. made revisions. All authors discussed the results and approved the final manuscript.

\section{Acknowledgments}

The authors would be grateful to Dr. Arsis Ahmadieh and Salar Samimi for providing the CT images. The study was not supported by any funding.

\section{Additional Information}

\section{Competing interests}

The authors declare no conflict of interest. 
Figures

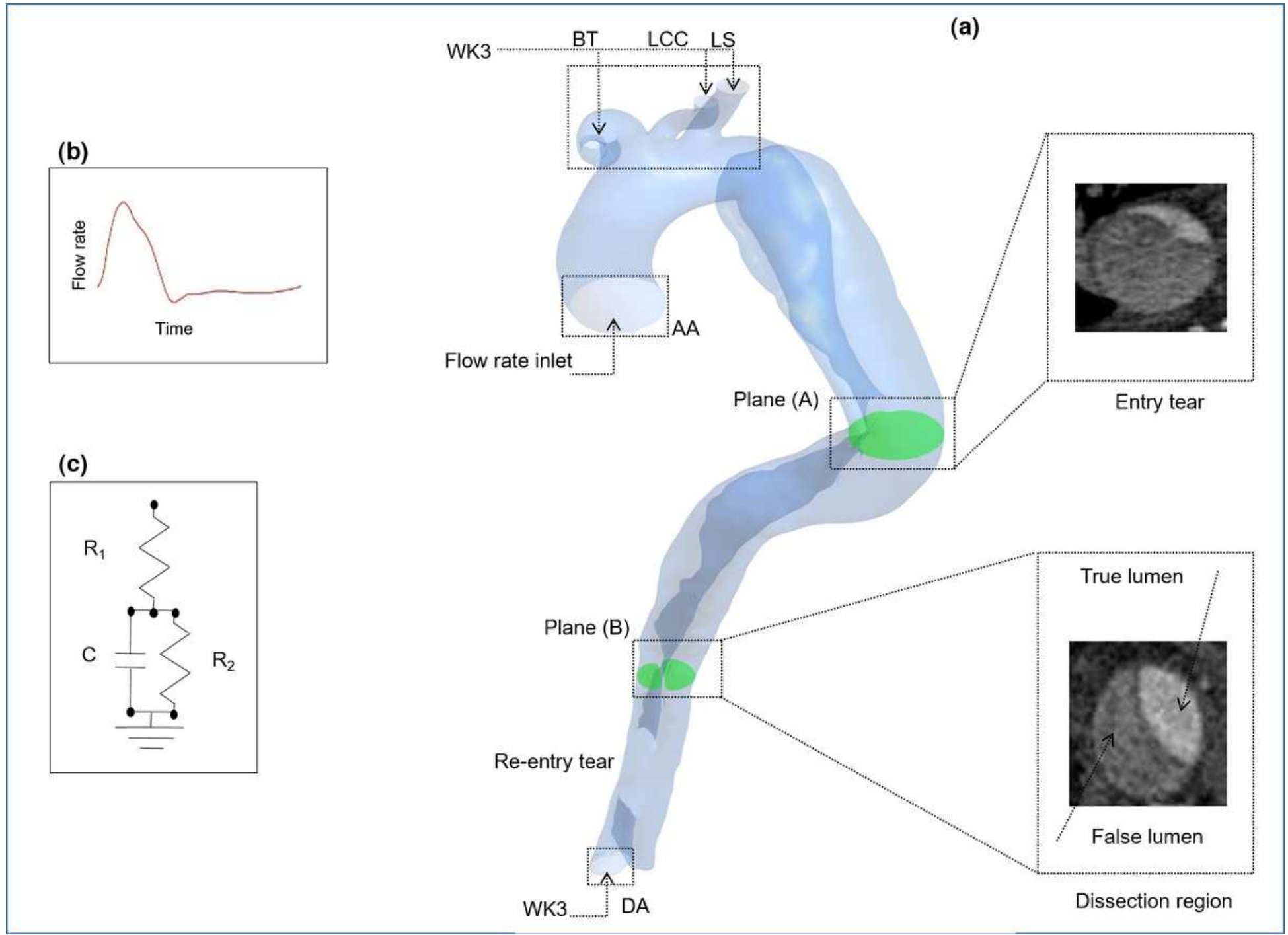

Figure 1

Geometry and boundary conditions. (a) Patient-specific dissected aorta geometry with two planes to illustrate the dissection region and entry tear. The boundaries are names with black arrows: DA: descending aorta, LS: left subclavian artery, AA: ascending aorta, LCC: left common carotid artery, BT: brachiocephalic trunk (b) Flow rate inlet diagram; (c) 3-element Windkessel boundary condition. 


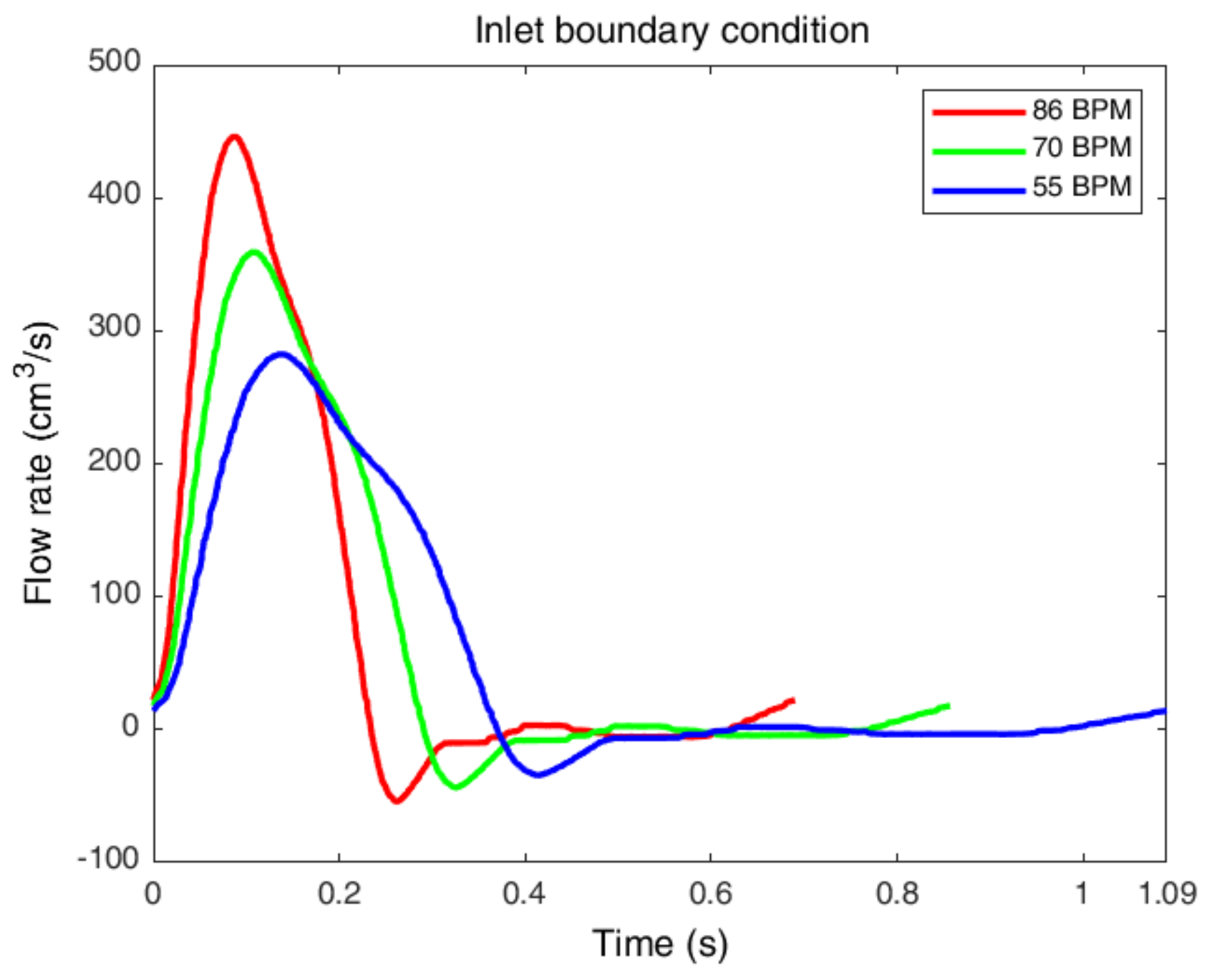

Figure 2

Input flow rate diagrams at the ascending aorta for all three heartbeats. 


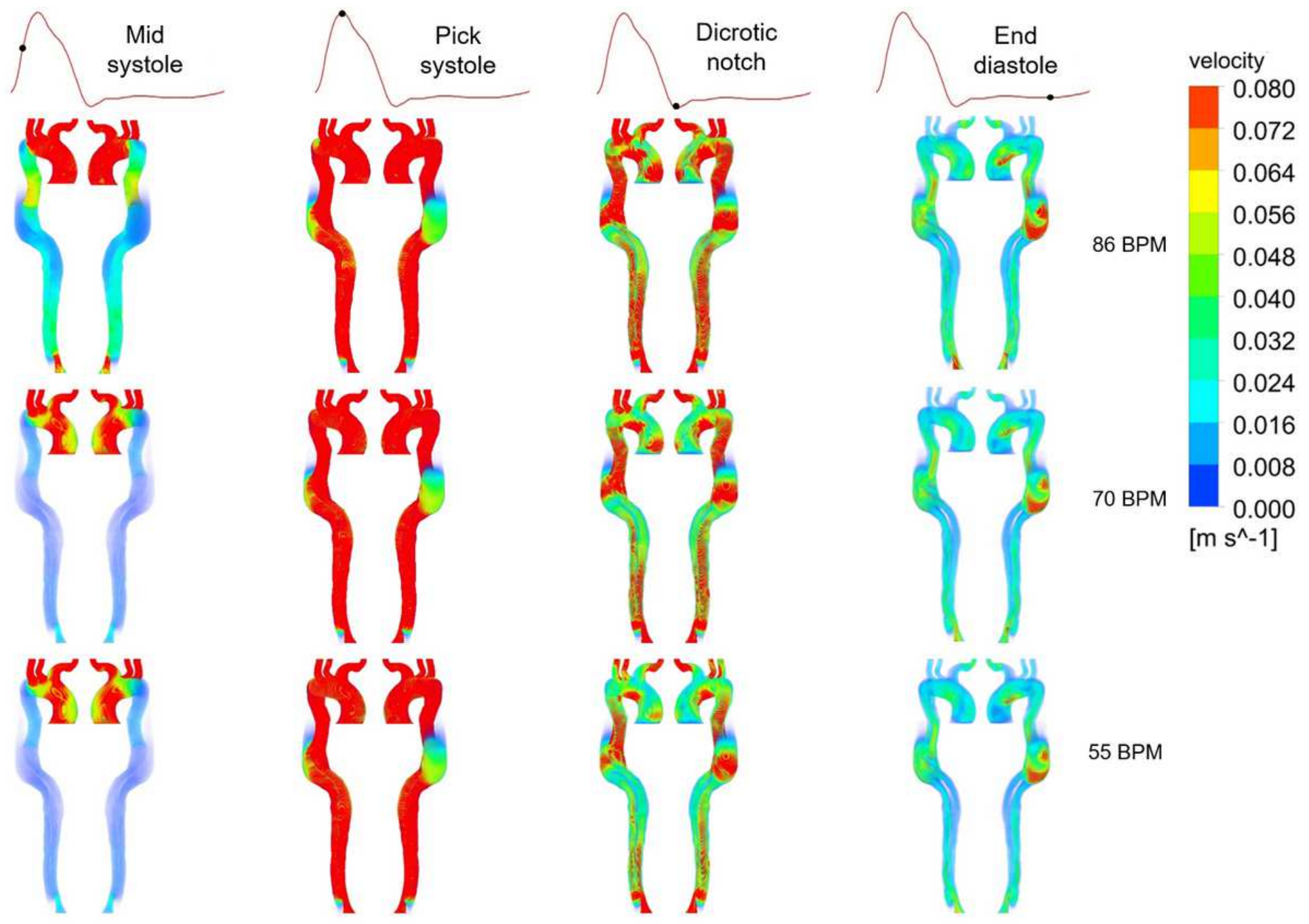

Figure 3

Velocity distribution for all three cases at four cardiac points. 


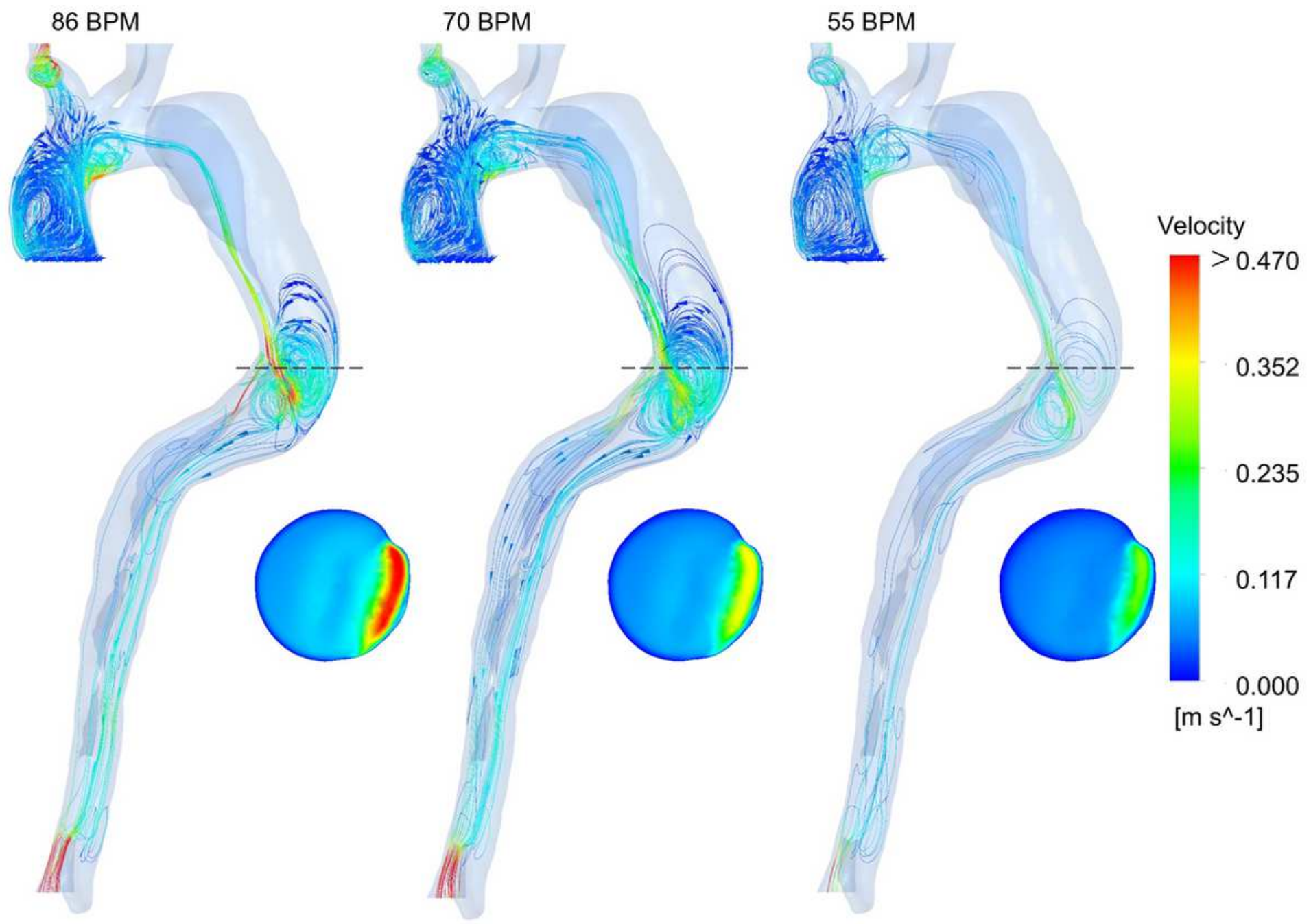

Figure 4

Velocity magnitude and streamlines for the three models at end-diastole. 


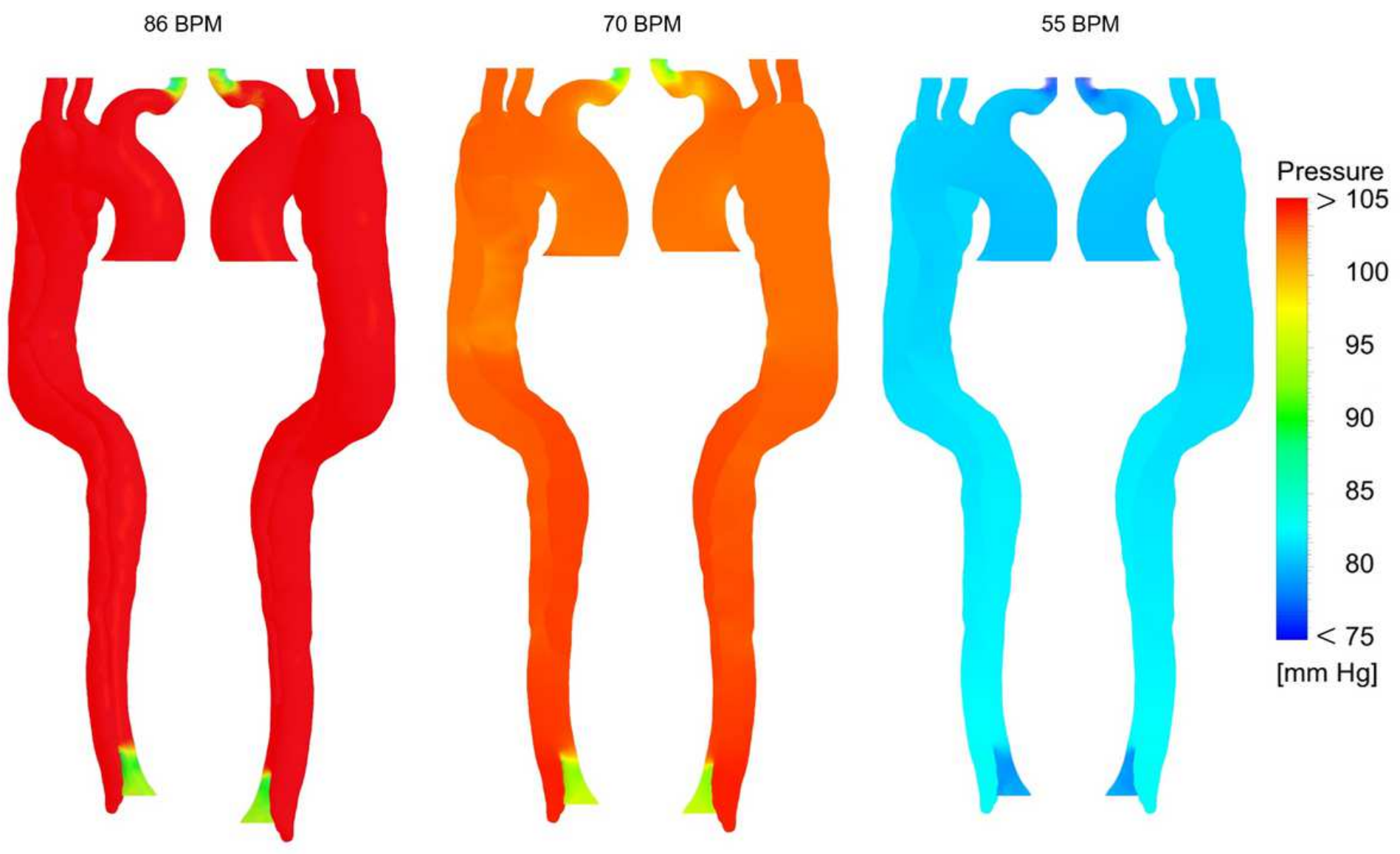

Figure 5

Pressure distribution for three cases at pick systolic blood pressure. 


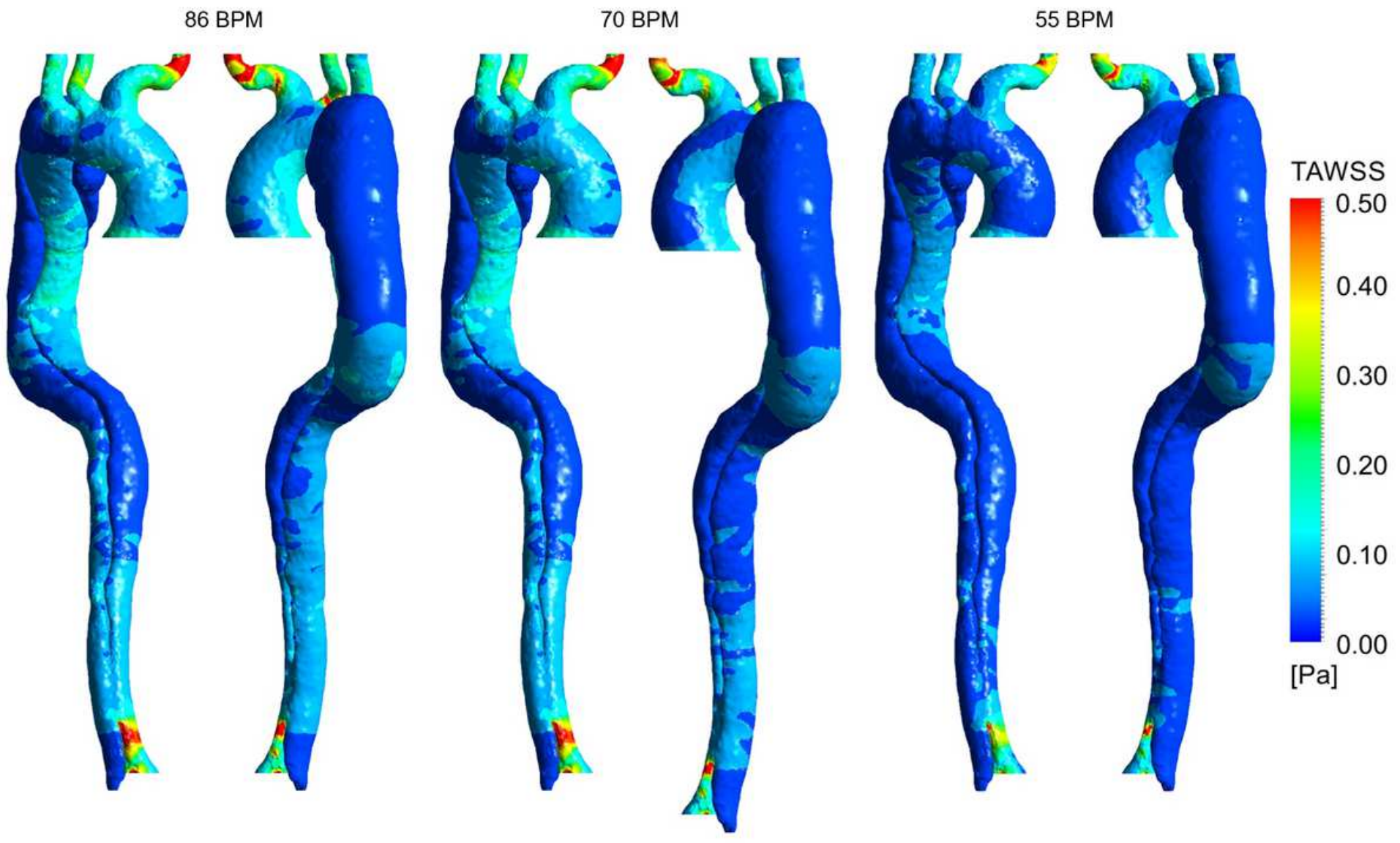

Figure 6

Time-averaged wall shear stress (TAWSS) distribution for three cases. 
(a)

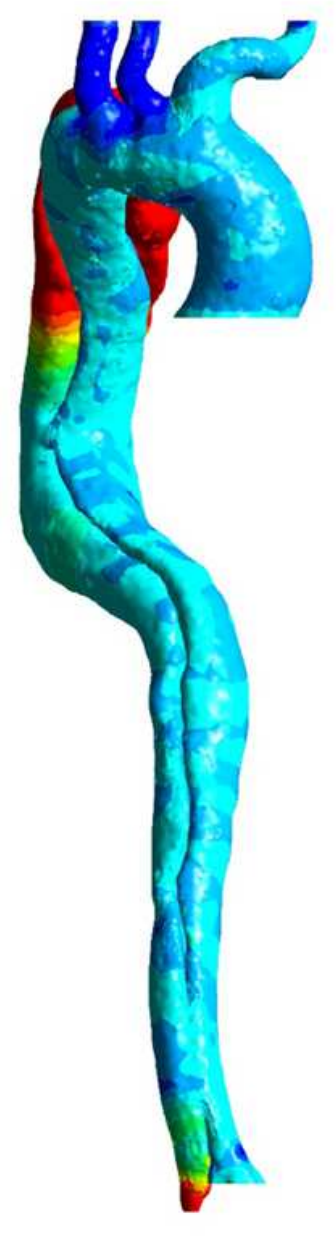

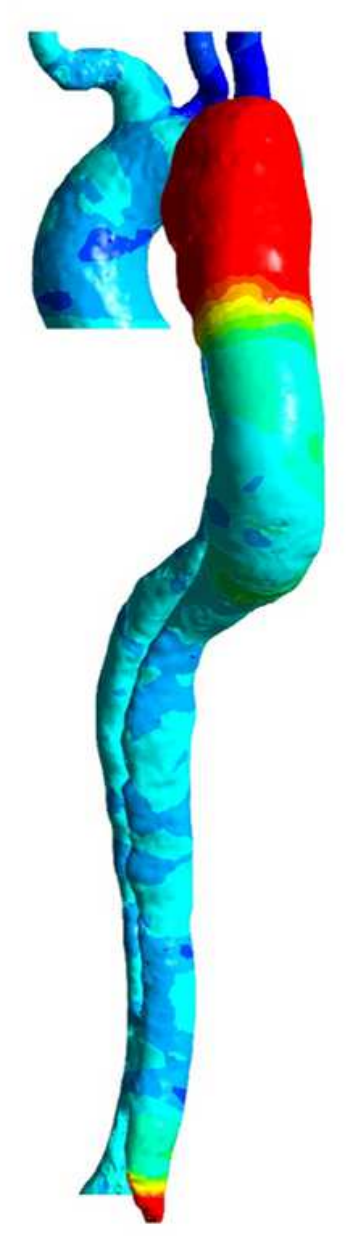

(b)
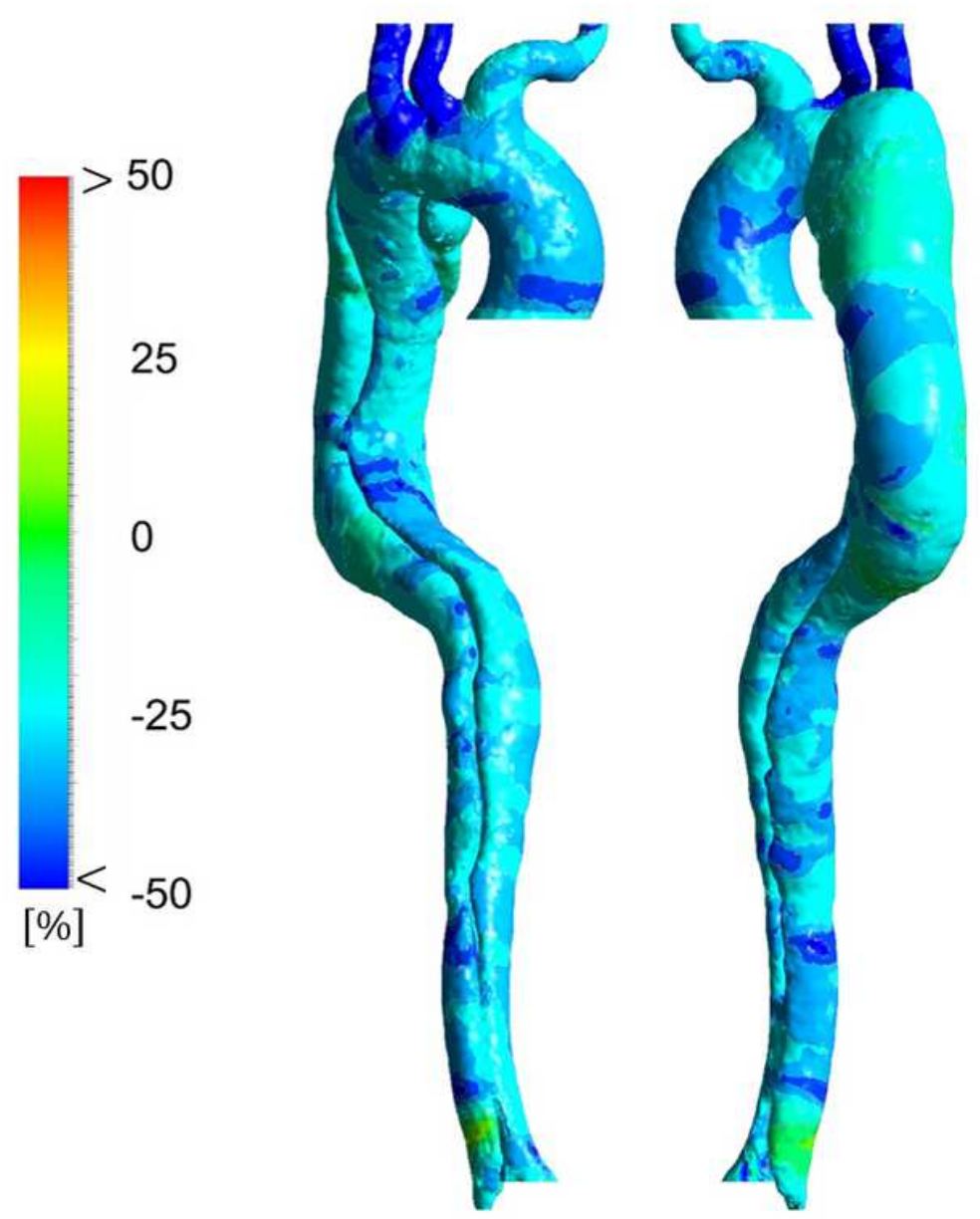

Figure 7

Percentage difference in time-averaged wall shear stress (TAWSS) relative to different heart rates. (a) Percentage difference in TAWSS between 86 BPM and 70 BPM; (b) Percentage difference in TAWSS between 55 BPM and 70 BPM. 


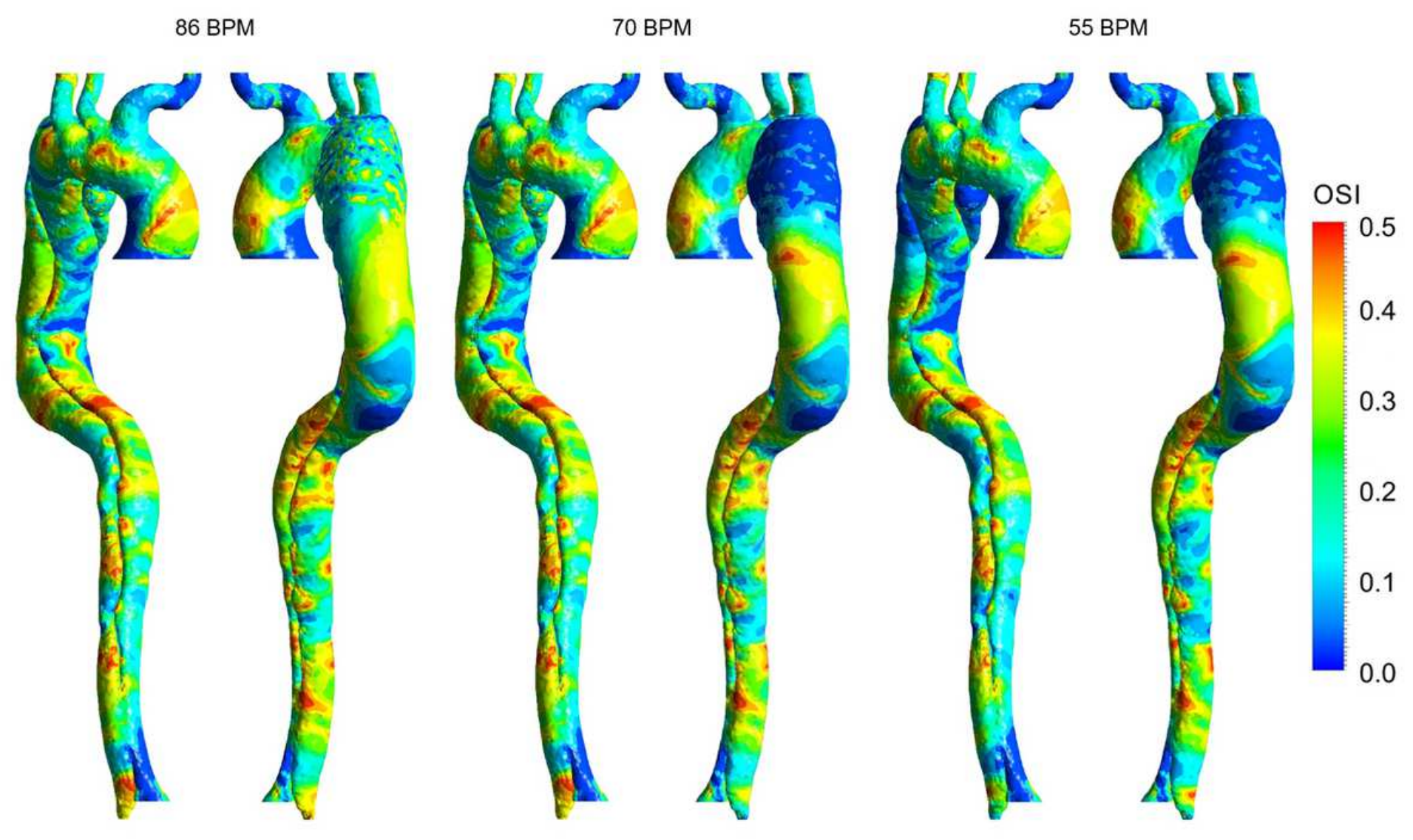

Figure 8

Oscillatory shear index (OSI) distribution for three cases. 
(a)

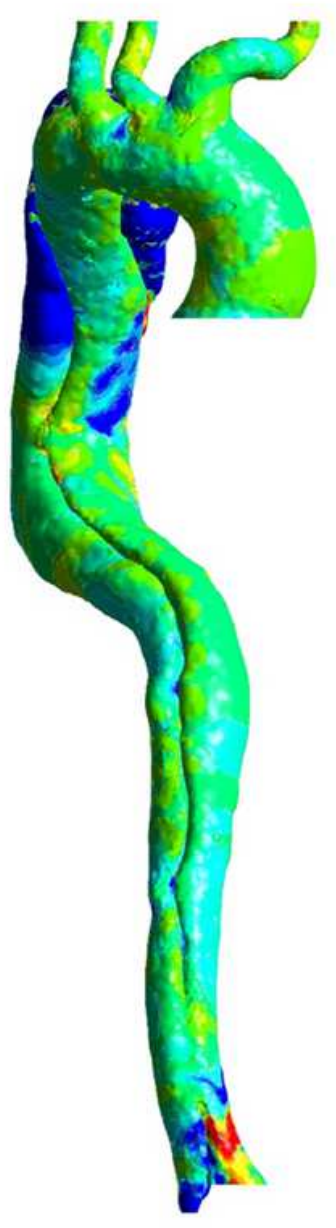

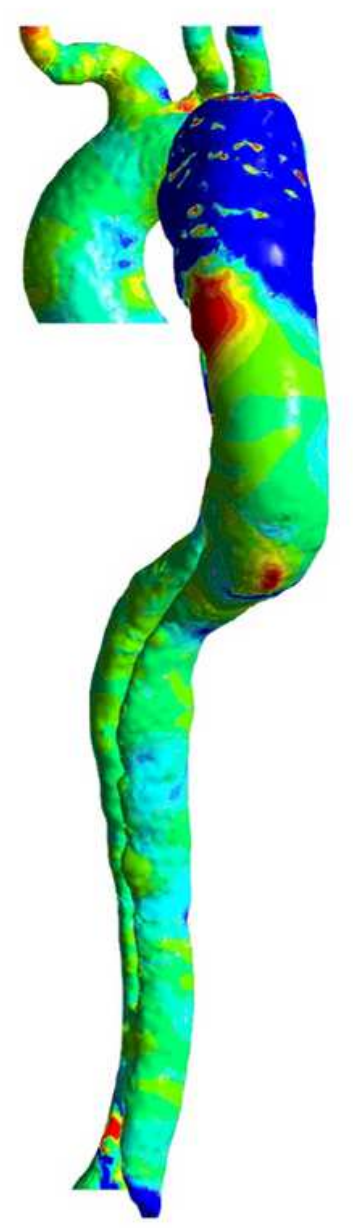

(b)
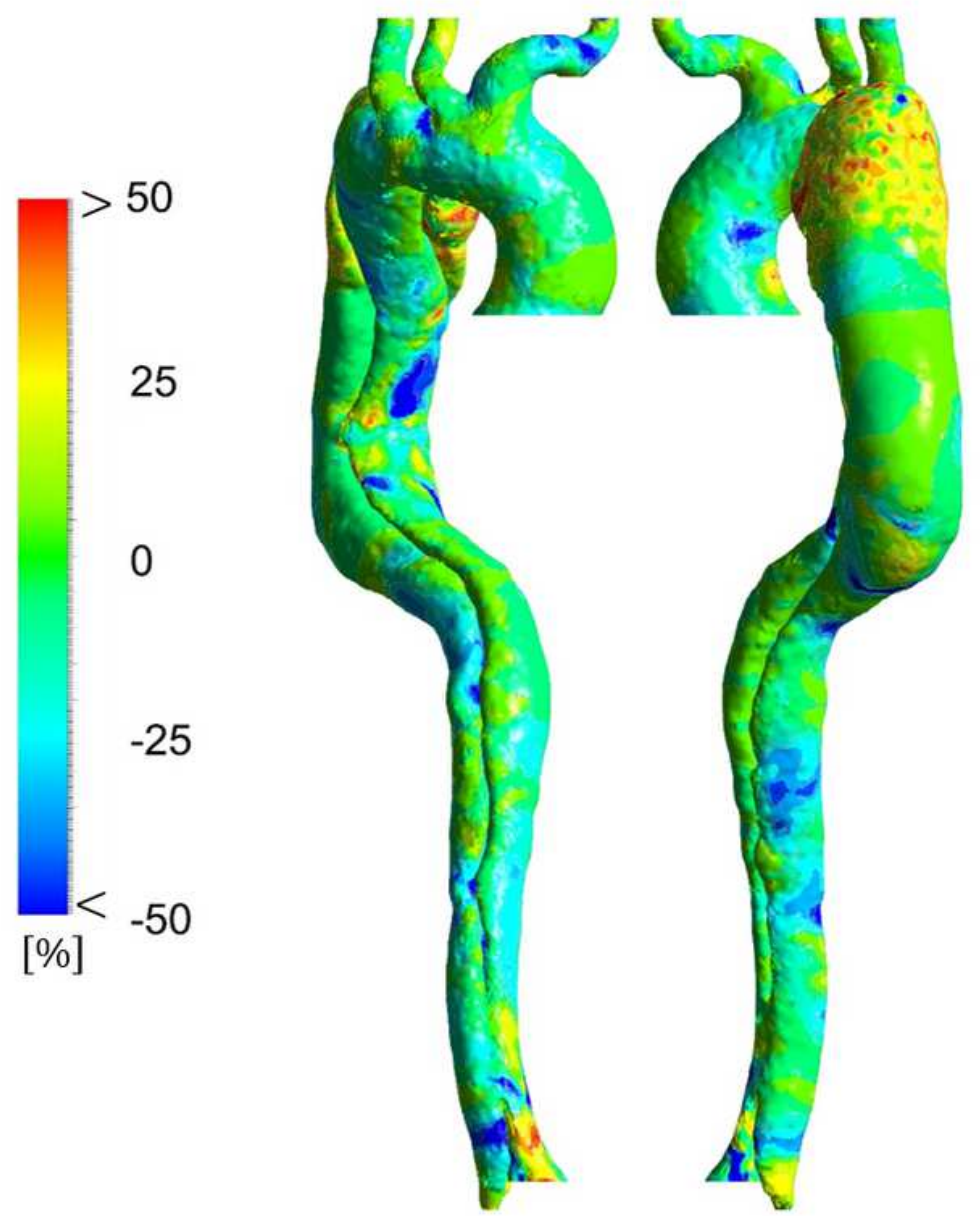

Figure 9

Percentage difference in oscillatory shear index (OSI) relative to different heart rates. (a) Percentage difference in OSI between 86 BPM and 70 BPM; (b) Percentage difference in OSI between 55 BPM and 70 BPM. 


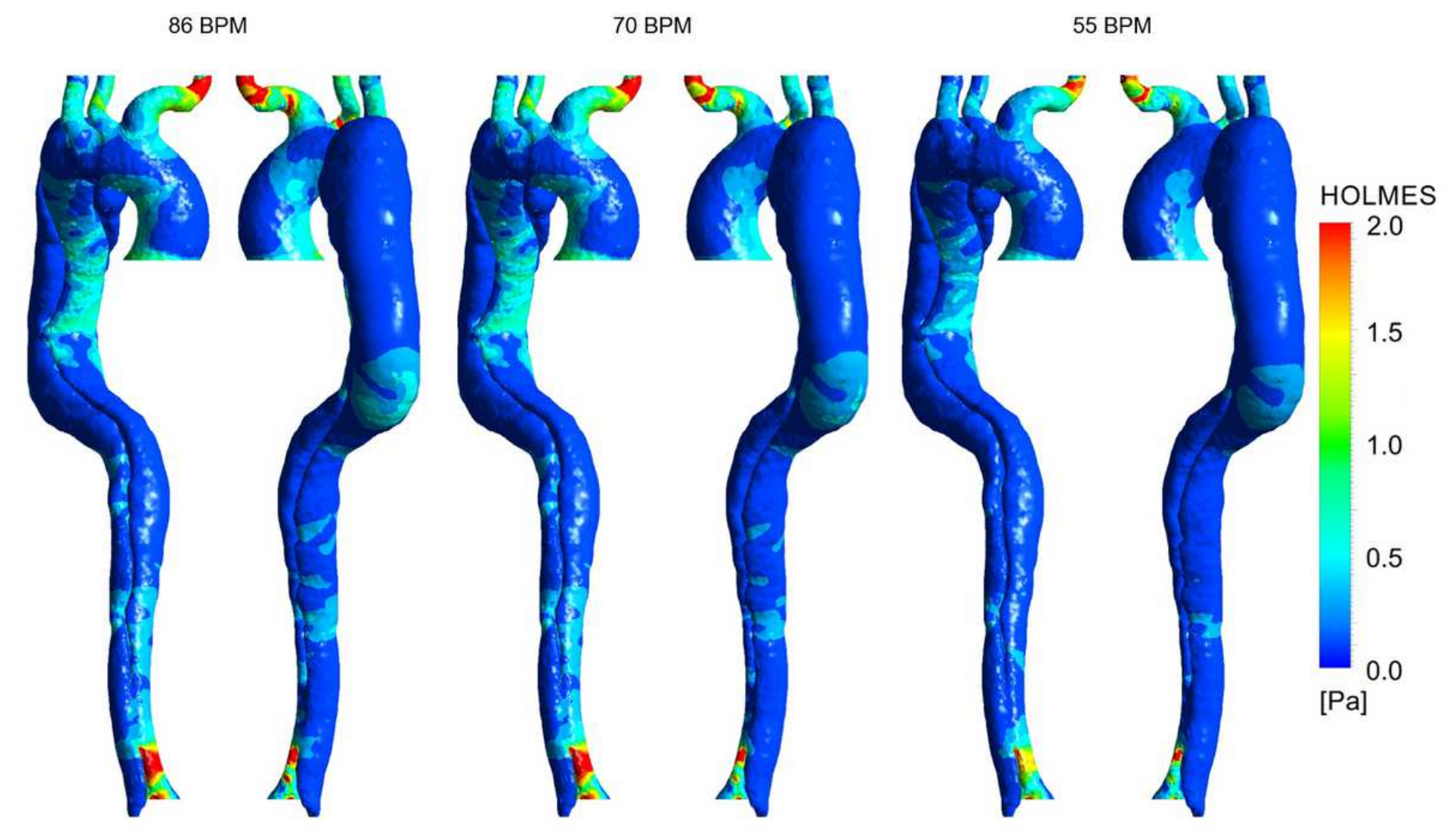

Figure 10

Highly oscillatory, low magnitude Shear (HOLMES) distribution for three cases. 
(a)

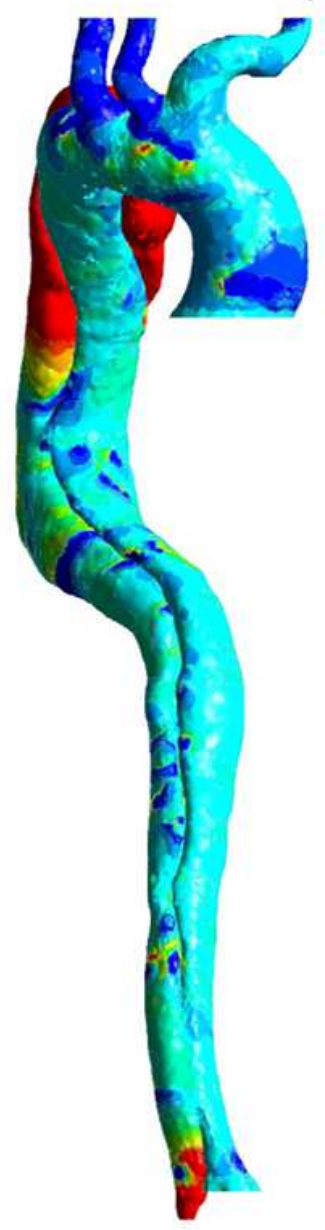

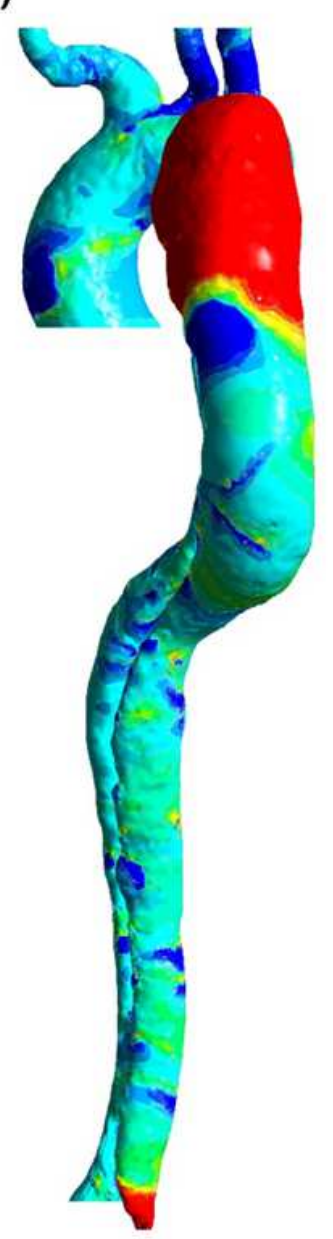

(b)
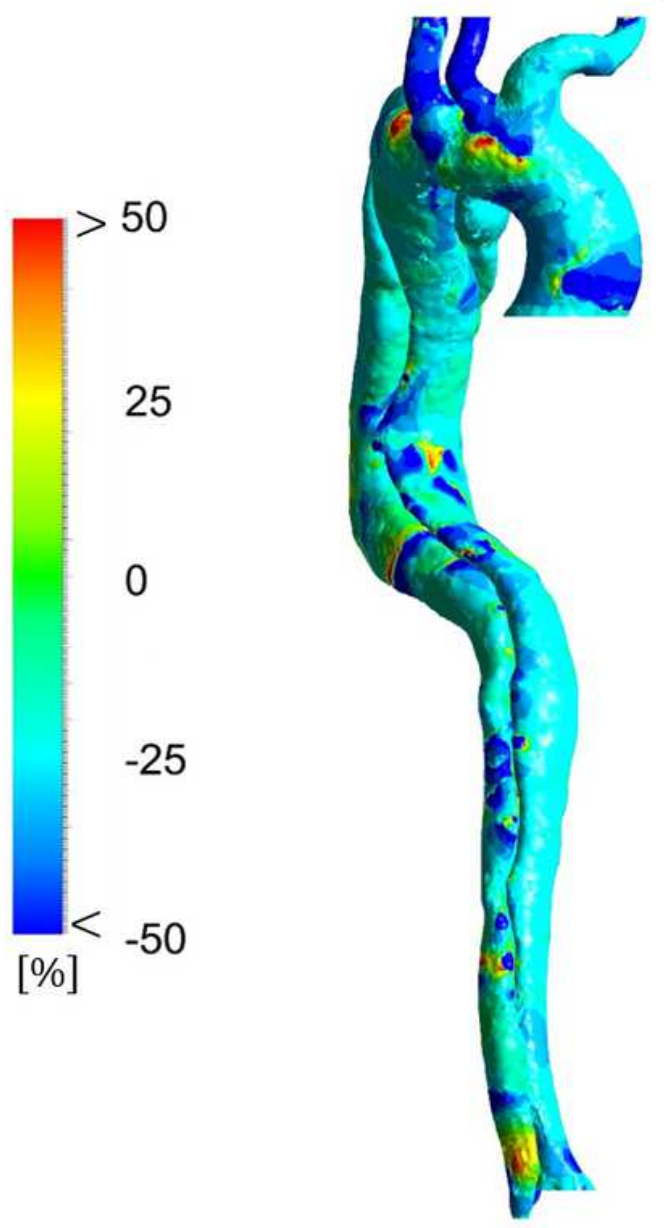

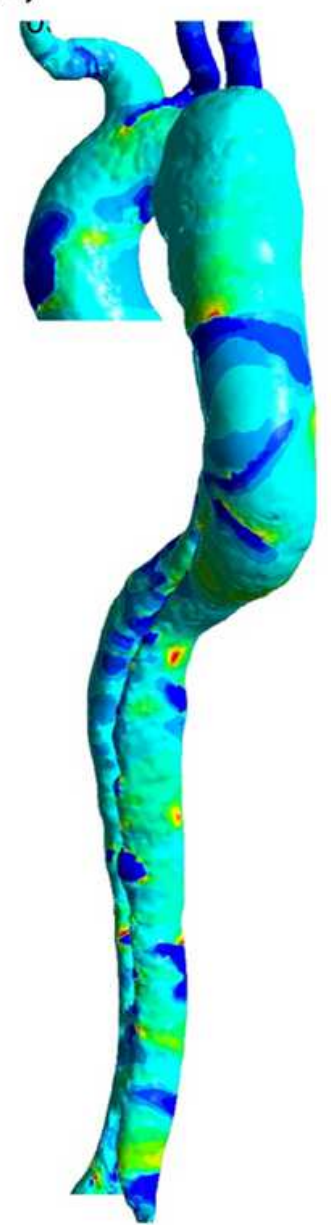

\section{Figure 11}

Percentage difference in highly oscillatory, low magnitude Shear (HOLMES) relative to different heart rates. (a) Percentage difference in HOLMES between 86 BPM and 70 BPM; (b) Percentage difference in HOLMES between 55 BPM and 70 BPM. 